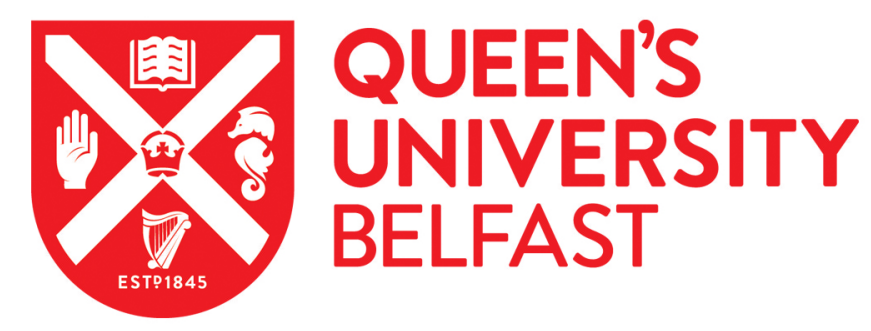

\title{
Nonlinear Aerodynamic and Aeroelastic Model Reduction using a Discrete Empirical Interpolation Method
}

Yao, W., \& Marques, S. (2017). Nonlinear Aerodynamic and Aeroelastic Model Reduction using a Discrete Empirical Interpolation Method. AIAA Journal, 55(2), 624-637. https://doi.org/10.2514/1.J055143

Published in:
AIAA Journal

Document Version:

Peer reviewed version

Queen's University Belfast - Research Portal:

Link to publication record in Queen's University Belfast Research Portal

Publisher rights

(C)2017 AIAA

This work is made available online in accordance with the publisher's policies.

\section{General rights}

Copyright for the publications made accessible via the Queen's University Belfast Research Portal is retained by the author(s) and / or other copyright owners and it is a condition of accessing these publications that users recognise and abide by the legal requirements associated with these rights.

Take down policy

The Research Portal is Queen's institutional repository that provides access to Queen's research output. Every effort has been made to ensure that content in the Research Portal does not infringe any person's rights, or applicable UK laws. If you discover content in the Research Portal that you believe breaches copyright or violates any law, please contact openaccess@qub.ac.uk. 


\section{Model reduction for nonlinear aerodynamics and}

\section{aeroelasticity using a}

\section{Discrete-Empirical-Interpolation Method}

W. YaoResearch Fellow, School of Mechanical and Aerospace Engineering, Queen's

University Belfast, now at National University of Singapore, and MAIAA and S. Marques

A novel surrogate model is proposed in lieu of Computational Fluid Dynamics (CFD) solvers, for fast nonlinear aerodynamic and aeroelastic modeling. A nonlinear function is identified on selected interpolation points by a discrete empirical interpolation method (DEIM). The flow field is then reconstructed using a least square approximation of the flow modes extracted by proper orthogonal decomposition (POD). The aeroelastic reduce order model (ROM) is completed by introducing a nonlinear mapping function between displacements and the DEIM points. The proposed model is investigated to predict the aerodynamic forces due to forced motions using a $N A C A 0012$ airfoil undergoing a prescribed pitching oscillation. To investigate aeroelastic problems at transonic conditions, a pitch/plunge airfoil and a cropped delta wing aeroelastic models are built using linear structural models. The presence of shock-waves triggers the appearance of limit cycle oscillations (LCO), which the model is able to predict. For all cases tested, the new ROM shows the ability to replicate the nonlinear aerodynamic forces, structural displacements and reconstruct the complete flow field with sufficient accuracy at a fraction of the cost of full order CFD model. 


\section{NOMENCLATURE}

$\mathbf{A} \quad=$ matrix of snapshots

$b, c \quad=$ aerofoil semi-chord and chord, respectively

$c_{i} \quad=$ radial basis function artificial neural network neuron $i$ center

$C_{L} \quad=$ lift coefficient

$C_{N} \quad=$ normal force coefficient

$C_{M} \quad=$ pitching moment coefficient

$\mathbf{f} \quad=$ fluid force acting on structure

$\mathbf{H}=$ radial basis function design matrix

$h \quad=$ plunge coordinate

$h_{i j} \quad=$ radial basis function for snapshot $i$ with respect to neuron $j$

$\mathbf{K}=$ structure stiffness matrix

$\mathbf{M}=$ structure mass matrix

$M_{\infty} \quad=$ free-stream Mach number

$M \quad=$ number of $\mathrm{POD}$ basis retained

$\mathbf{P} \quad=$ interpolation indices matrix

$n \quad=$ number of delayed displacements recorded

$q \quad=$ dynamic pressure

$\mathbf{R}=$ vector of fluid and structural equations residuals

$r_{i} \quad=$ radial basis function artificial neural network neuron $i$ radius

$\mathbf{S}=$ diagonal matrix containing singular values

$t \quad=$ time

$\mathbf{T} \quad=$ matrix of POD basis

$U_{\infty}, \mathbf{U}=$ free-stream velocity, velocity vector, respectively

$\mathbf{u} \quad=$ variable for model reduction process

$V_{s} \quad=$ reduced velocity index

$\mathbf{w} \quad=$ vector of fluid and structural unknowns

$\mathbf{x} \quad=$ list of displacements

$\alpha \quad=$ angle of attack

$\mathbf{\Phi} \quad=$ radial basis function artificial neural metwork weights vector

$\omega, \kappa=$ frequency and reduced frequency, $\left(\frac{2 \omega}{U_{\infty} c}\right)$

$\varphi \quad=$ phase angle

$\rho \quad=$ fluid density 


\section{Introduction}

Despite the ever increasing computing power available, high computational cost still prevents state-of-art CFD to be routinely used in procedures where iterative processes are intrinsic, such as design optimization, combustion, uncertainty quantification, and more pertinent to this work: dynamic aeroelasticity.

There has been a continuous interest in developing efficient and reliable techniques able to overcome the need for full blown time-accurate CFD simulations. In the context of dynamic aeroelasticity, several advances have been made. If the Jacobians of the CFD-CSD (Computational Structural Dynamics) system are available, it is possible to determine the stability of the dynamic system (i.e. flutter boundary) without resorting to time-domain simulations by eigenvalue analysis[1], in addition a nonlinear reduced-order model (ROM) can be built to predict the post-critical behaviour, i.e. LCO, by further manipulating the aeroelastic critical eigenvector[2]. The prediction of LCO can also be achieved by exploiting the periodicity of the aeroelastic system using the so called harmonic-balance method[3, 4]. The application of the aforementioned methods requires significant modifications to existing codes. To limit any potential code modifications, surrogate models based on samples or snapshots from high-fidelity analysis can be built. Several techniques have been proposed for linear modeling, or to exploit linearizations of the original CFD system: system identification (ID) or modal superimposition methods such as Volterra Series[5, 6], POD[7-10] and Balanced Proper Orthogonal Decomposition[11, 12]. A linear ROM can only be used in flows where nonlinearities can be neglected safely, such as the onset of flutter.

Building a nonlinear ROM poses a more significant challenge and is the subject of much attention within the modeling community. Hence, several nonlinear ROMs have been developed and applied successfully as nonlinear surrogates, for example: Artificial Neural Network Auto-Regressive Exogenous (ANN-ARX)[13, 14], Kriging-ARX[15], Continuous Time Recurrent Neural Network[16]. The nonlinear models proposed in references[14-16] are built using the pitching angle or structural displacements as input and the aerodynamic force as output, however the models are not able to provide flow field information, such as pressure. Fagley et al. used POD and ANN-ARX to investigate the free shear layer transient effects[13]. Following the same idea, Lindhorst et al. employed 
POD with a Markov-Chain interpolator function and Radial-Basis Function Artificial Neural Network (RBF-ANN) to model nonlinear aerodynamic flows for aero-structural calculations, in this approach the input and output were the POD coefficients of the grid displacement and aerodynamic force distribution $[17,18]$, the authors also suggested that excessive POD modes may degrade the approximation accuracy. Therefore, it is cumbersome to balance the number of POD modes for both input and output. The Discrete Empirical Interpolation Method (DEIM), proposed by Chaturantabut et al. [19], approximates the full order state vector in a least square sense, with POD modes through a small number of interpolation points. DEIM requires a nonlinear system representation in matrix-vector form a priori to build the ROM. Following the DEIM concepts, Carlberg et al. [20] proposed the Gauss-Newton with approximated tensors (GNAT) method to construct a nonlinear ROM for computing separated, unsteady flows. GNAT implements Gauss-Newton methods to solve the nonlinear problem, and requires the evaluation of the Jacobian (a nonlinear residual over the solution vector), which is computationally expensive in practice and renders it less attractive for aeroelastic simulations. Eventually, only a reduced or small matrix is iteration dependent to capture the nonlinearities.

Several of these techniques have been demonstrated to construct nonlinear ROM or surrogate models for aeroleasticity successfully[2, 6, 14, 16-18]; the majority of these have been investigated to provide a nonlinear mapping function for dynamic aeroelastic problems such as LCOs, but at the expense of flow field information. Recently, Yao and Liou[21] proposed a nonlinear model by assembling a set of linear models that are each valid in the vicinity of sampling points, which provides not only the aerodynamic force but also the flow field information for nonlinear aeroelastic modeling.

The aim of this work is to extend and validate the POD/DEIM approach to nonlinear aeroelastic problems, while retaining the ability to reconstruct the flow field from snapshots of the full order state vector. The new ROM uses the POD/DEIM approach to reduce the order of the fluid system and an RBF-ANN to provide the nonlinear mapping between structural displacements and the flow variable of interest. The DEIM determines the optimum interpolation points to, crucially, reconstruct the flow field; the same points are used for training the RBF-ANN. With the mapping 
function built, the link between the RBF-ANN and the DEIM allows the reconstruction of the complete flow field online and the calculation of the aerodynamic forces.

In the next sections, the paper gives an overview of the CFD solver used, this is followed by a detailed presentation of the POD/DEIM framework used to reduce the dimension of the CFD system and the method to employ an RBF-ANN and DEIM to perform aeroelastic analysis is outlined. The capabilities of the ROM are illustrated through three test cases, including a 2D airfoil forced motion problem and two additional examples of free to vibrate nonlinear aeroelastic systems: a pitch/plunge airfoil and a cropped delta wing. Finally, results are reviewed in light of their accuracy and computational cost and overall conclusions are presented.

\section{Formulation}

The overall strategy is described next. As discussed in the introduction, the purpose of the ROM is to reduce the effort associated with high-fidelity unsteady aerodynamic calculations required for aeroelastic predictions. Typical CFD/CSD solvers exchange information in the form of displacements, velocities and pressures, hence the point of the surrogate model is to provide the surface pressure (or equivalent) associated with any translations or rotations from the structure. For that purpose, it is possible to define a nonlinear mapping between inputs and outputs that can replace the discrete full order CFD/CSD system[22]:

$$
\mathbf{Q}_{f}(t)=\mathcal{F}\left(\mathbf{w}_{s}(t), \mathbf{w}_{s}(t-\Delta t), \mathbf{w}_{s}(t-2 \Delta t), \ldots, \mathbf{w}_{s}(t-n \Delta t)\right)
$$

where $\mathbf{Q}_{f}$ represents fluid variables such as pressure or temperature; $\mathbf{w}_{\mathbf{s}}=[\boldsymbol{\xi}, \dot{\boldsymbol{\xi}}]$ are the structural displacement and velocity. The inputs to the nonlinear function $\mathcal{F}$, is the structural displacement and its delay and the fluid variables are the output. In practice, the surrogate only approximates $\mathcal{F}$. To build the surrogate, the following steps are required:

1. generate an adequate training trajectory covering the parameter space of interest;

2. collect snapshots along the training trajectory of the full-order model (FOM) response;

3. compute POD modes;

4. apply DEIM to POD modes and determine interpolation points; 
5. build RBF-ANN for each interpolation point;

then, using the RBF-ANN and designated interpolation points, it is possible to reconstruct the whole flow field using the DEIM and to integrate the surface pressure to determine the aerodynamic loads for the structural solver.

\section{A. Time-Domain Fluid-Structural Solver}

To obtain the snapshots required to build the ROM, a research CFD-FEM (Finite Element Model) code is used. The aerodynamic model is based on the Euler equations for fluid flow and as is typical in computational aeroelasticity a modal structural model is used to represent the structure. Consider the semi-discrete form of the coupled system of conservation laws described as:

$$
\frac{d \mathbf{w}}{d t}=-\mathbf{R}(\mathbf{w})
$$

where

$$
\mathbf{w}=\left[\mathbf{w}_{f}, \mathbf{w}_{s}\right]^{T}
$$

and

$$
\mathbf{R}=\left[\mathbf{R}_{f}, \mathbf{R}_{s}\right]^{T}
$$

here $\mathbf{w}_{f}$ is the vector of the fluid conserved variables $(\rho, \rho \mathbf{U}, \rho E)$ and $\mathbf{R}_{f}$ is the residual which contains the discretization of the convective terms of the equations, given by the $A U S M^{+}-u p$ flux function[23]; a MUSCL scheme together with the Van Albada limiter is employed to achieve $2^{\text {nd }}$ order spatial accuracy in smooth regions[24]. The scheme is marched forward in time using a dual-time-stepping strategy, four-stage Runge-Kutta scheme[4]. $\mathbf{R}_{s}$ is the residual of the structural equations transformed into a state-space representation, involving the generalized aerodynamic forces from the fluid system[4, 25]. For wing structures, the aerodynamic loads and structural displacements transfers between the CFD/CSD models are obtained through the Infinite Plate Spline (IPS) method. Transfinite Interpolation is used to deform the CFD mesh and grid velocities are approximated using finite-differences. 


\section{B. Proper Orthogonal Decomposition}

The use of POD as a means of reducing the complexity of large dynamic systems described by partial-differential equations extends to several engineering applications.[26] In this work, the POD modes (also referred to as POD basis) of the CFD system are sought. For a set of snapshots of the flow field, $\mathbf{A}=\left[\mathbf{u}_{1}, \mathbf{u}_{2}, \ldots, \mathbf{u}_{n_{s}}\right]$, the POD basis represents an optimal set of orthogonal vectors that can best approximate the snapshots. The snapshots are obtained from the time-accurate CFD/CSD solution and are recorded at specified time instances. For the current applications, only the pressure field is recorded, hence each snapshot is a vector $\mathbf{u} \in \mathbb{R}^{n_{c} \times 1}$, where $n_{c}$ is the number of cells in the CFD mesh, making $\mathbf{A} \in \mathbb{R}^{n_{c} \times n_{s}}$. A well known method to obtain the POD basis of $\mathbf{A}$ is to compute its left singular vectors by singular value decomposition (SVD), however for large systems where $n_{s}<n_{c}$, then the SVD is applied to $\mathbf{A}^{T} \mathbf{A} \in \mathbb{R}^{n_{s} \times n_{s}}$ :

$$
\mathbf{A}^{T} \mathbf{A}=\mathbf{T S V}^{T}
$$

where $\mathbf{S}=\operatorname{diag}\left[\lambda_{1}, \lambda_{2}, \ldots, \lambda_{n_{s}}\right]$ is the eigenvalue matrix and $\mathbf{T}, \mathbf{V}$ are the left and right singular vectors, respectively. The POD basis, $\mathbf{T}_{M} \in \mathbb{R}^{n_{c} \times M}$, is obtained from Eq. (6) with proper truncation according to the energy rank represented by the amplitude of the first $M$ eigenvalues, $\mathbf{S}_{M}=\operatorname{diag}\left[\lambda_{1}, \lambda_{2}, \ldots, \lambda_{M}\right]$, where $\lambda_{1}>\lambda_{2}>\ldots>\lambda_{M}$ and $M \ll n_{c}$.

$$
\mathbf{T}_{M}=\mathbf{A V S}_{M}^{-1 / 2}
$$

\section{Discrete Empirical Interpolation Method}

In this section, the DEIM is reviewed. The application of POD is limited to linear or mildly nonlinear time or parameter dependent system of partial-differential equations[27]. As nonlinearities become dominant, its efficiency deteriorates, rendering the use of POD unattractive for nonlinear aeroelastic problems. In this paper DEIM is used to overcome this limitation. In essence DEIM approximates a parametric nonlinear function, $\mathbf{u}(t)$, by projecting it onto the subspace spanned by the basis $\mathbf{T}_{M}$ as:

$$
\mathbf{u}(t) \approx \mathbf{T}_{M} \mathbf{c}(t)
$$


where $\mathbf{c}(t) \in \mathbb{R}^{M \times 1}$ are the corresponding coefficients. The methodology proposed by Chaturantabut et al. enables selecting $M$ distinct rows or interpolation indices so that the coefficients $\mathbf{c}(t)$ can be solved uniquely as described in algorithm 1[19]. The interpolation indices are defined by the matrix $\mathbf{P} \in \mathbb{R}^{n_{c} \times M}$, where each row contains interpolation indices, which leads to

$$
\mathbf{P}^{T} \mathbf{u}(t)=\mathbf{P}^{T} \mathbf{T}_{M} \mathbf{c}(t)
$$

Substituting Eq. 8 into Eq. 7, obtain:

$$
\mathbf{u}(t) \approx \mathbf{T}_{M}\left(\mathbf{P}^{T} \mathbf{T}_{M}\right)^{-1} \mathbf{P}^{T} \hat{\mathbf{u}}(t)
$$

where $\hat{\mathbf{u}}(t)$ contains the components corresponding to non-zeros indices in $\mathbf{P}$, hence Eq.9 shows that $M$ elements are required to recover the full order vector $\mathbf{u}(t)$. For fluid systems, let $\mathbf{u}(t)$ represent the pressure field. As described in Eq. 1, the nonlinear mapping function $\mathcal{F}$ can be constructed at $M$ interpolation points. The full order vector $\mathbf{u}(t)$ is reconstructed by Eq. 9. The DEIM algorithm to determine the interpolation indices is repeated below for completeness.

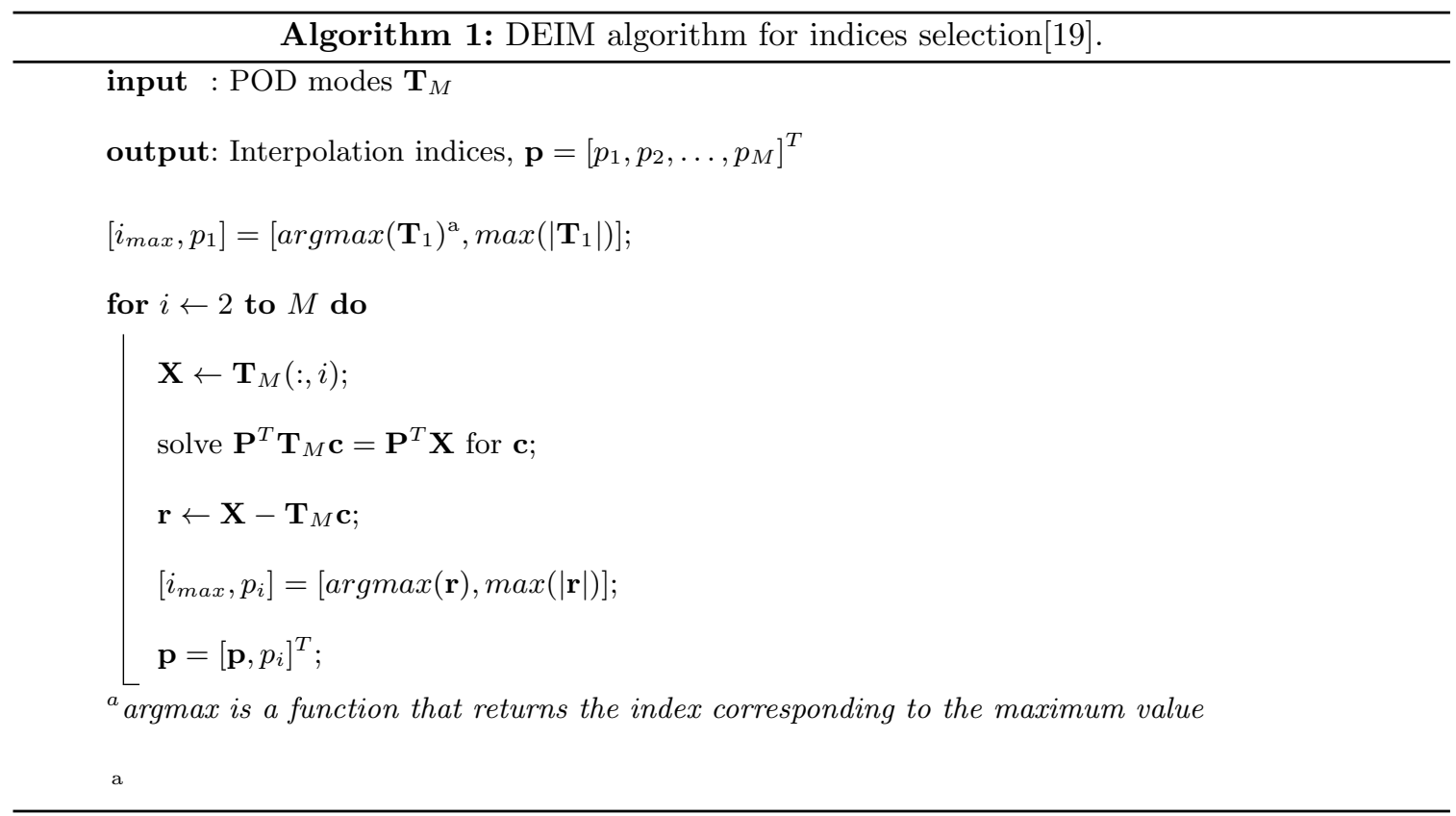

\section{Radial Basis Function - Artificial Neural Network}

The nonlinear mapping function in Eq. 1 is defined by an RBF-ANN, depicted in Figure 1. This approach is considered as a multi-input and multi-output (MIMO) nonlinear system identification 
method. The input/output is at user's discretion according to the specific physical problem at hand. In the present paper, the input and output are displacements and pressures from the structure and fluid, respectively. The idea is to construct the RBF-ANN of the pressure at $M$ interpolation points defined by the algorithm 1 . The neuron is modeled by RBFs as[28]

$$
h_{i}=e^{-\frac{\left|x(t)-c_{i}\right|^{2}}{r_{i}}}, i=1,2, \ldots, M
$$

where $c_{i}$ and $r_{i}$ are the $i$ th neuron center and radius, respectively; $\mathbf{x}$ is the input, given by

$$
\mathbf{x}(t)=[\boldsymbol{\xi}(t), \boldsymbol{\xi}(t-\Delta t), \ldots, \boldsymbol{\xi}(t-n \Delta t)]
$$

Many approaches are available to determine the RBF center $c_{i}$, such as K-means, or even evolving input data. In this work even evolving input data is chosen to provide the RBF center $c_{i}$, due to its simplicity. The radius $r_{i}$ or width is defined as per reference[17]:

$$
r_{i}=\frac{1}{M} \sum_{j=1}^{M}\left\|c_{j}-c_{i}\right\|^{2}, i=1,2, \ldots, M
$$

Once $c_{i}$ and $r_{i}$ are identified, the training process can be completed. The mapping relation between inputs and outputs is obtained by a weighted sum; the function weights $\boldsymbol{\Phi}$ are obtained using the target flow variables defined by $\hat{\mathbf{u}}$, at the interpolation points:

$$
\hat{\mathbf{u}}=\mathbf{H} \Phi
$$

where

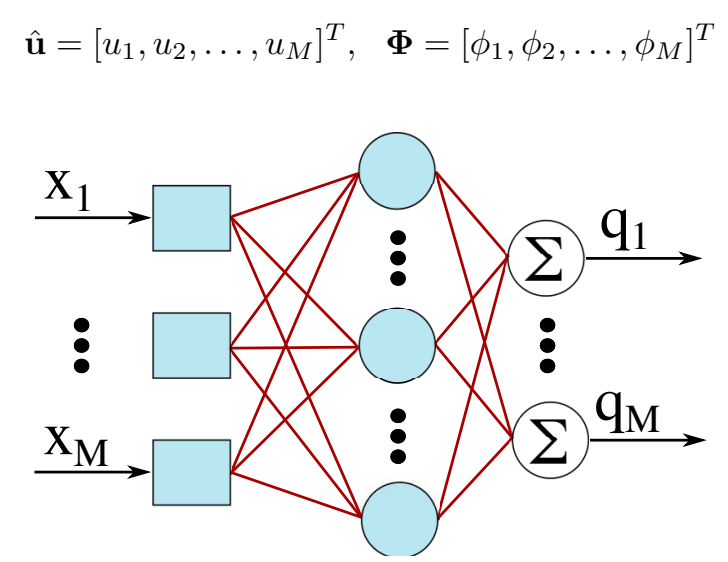

Fig. 1 RBF-ANN Schematic. 
and

$$
\mathbf{H}=\left[\begin{array}{ccc}
h_{11} & \ldots & h_{1 M} \\
\vdots & \ddots & \vdots \\
h_{M 1} & \ldots & h_{M M}
\end{array}\right]
$$

The weight matrix $\boldsymbol{\Phi}$ can be solved as,

$$
\Phi=\mathbf{H}^{+} \hat{\mathbf{u}}
$$

$\boldsymbol{\Phi}^{+}$is Moore-Penrose pseudo inverse, which is defined as,

$$
\mathbf{H}^{+}=\left(\mathbf{H}^{T} \mathbf{H}\right)^{-1} \mathbf{H}^{T}
$$

To avoid possible over fitting and instabilities, a weight penalty or a regularization parameter $\varepsilon$ is introduced that is given by[28]:

$$
\mathbf{H}^{+}=\left(\mathbf{H}^{T} \mathbf{H}+\varepsilon \mathbf{I}\right)^{-1} \mathbf{H}^{T}
$$

the parameter $\varepsilon$ is a small number, however, it has no optimal value, and is problem dependent. Lindhorst et al. suggest $\varepsilon=10^{-7}$ is a reasonable choice for the NLR 7301 airfoil[17]. The two and three dimensional cases presented below also adopt the value $\varepsilon=10^{-7}$.

\section{Results}

\section{A. Test Case 1: AGARD CT-5}

The AGARD CT-5 test case consists of a pitching airfoil (NACA 0012) around the quarter chord[29]. The flow condition and motion parameters are detailed in Table 1 and the pitching motion is given by

$$
\alpha(t)=\alpha_{m}+\alpha_{0} \sin 2 \kappa t
$$

The grid convergence study from reference[4] has shown that an O-type grid with $61 \times 21$ points, in the circumferential and radial directions respectively, is adequate for this case. The training process aims to cover a range of pitching frequencies $\kappa$ and pitching amplitudes $\alpha_{0}$. It should be noted that there is no optimal procedure to generate training trajectories. Glaz et al. proposed 
the training trajectory to be generated by superimposition of multiple harmonics with different frequencies, amplitudes and phase angles, which as been shown to capture aerodynamic responses due to arbitrary and harmonic motions[15], it is defined as:

$$
\alpha_{0}=\sum_{i=1}^{n_{h}} \alpha_{i} \sin \left(2 \kappa_{i} t+\varphi_{i}\right)
$$

where, $n_{h}$ is the number of harmonics. The general guidance to derive a suitable parameter set is that the training database covers all regions of the input parameter space, which can be translated as a space-filling problem[15, 17], Latin Hypercube Designs offer a straightforward solution to this kind of problem. Therefore, in this case the parameter set $\left[\alpha_{i}, \kappa_{i}, \varphi_{i}\right]$ is obtained by sampling a uniform distribution using a Latin Hypercube sampling method. It is at the user's discretion to define the lower and upper bounds to generate the training trajectory of interest. Figure 2 shows the final training trajectory obtained using the following limits:

$$
\begin{gathered}
0^{\circ} \leq \alpha_{i} \leq 0.2^{\circ} \\
0.85 \kappa_{m} \leq \kappa_{i} \leq 1.15 \kappa_{m} \\
0 \leq \varphi_{i} \leq \pi
\end{gathered}
$$

The training trajectory contains multiple amplitudes and covers a range of frequencies as evidenced by Figure 2. It is important to note that $\alpha_{i}$ varies within is a relatively small range in Eq. (21), however the final training trajectory amplitude is one order of magnitude larger, this is a result of the superimposition process. The user can choose the bounds in Eq. (21)-(23) to cover the range of amplitudes $\alpha_{0}$ and frequencies $\kappa$ of interest.

Taking the training trajectory as the input, the pressure field snapshots are collected from each physical time instant, resulting in 1000 snapshots. Then POD modes are extracted using Eq. (6). As shown by Figure 3, the first POD mode represents the mean flow, and the second and third are associated with shock movement. The eigenvalue of each POD mode represents the energy rank,

\begin{tabular}{cccccc} 
Case & $M_{\infty}$ & $\alpha_{m}$ & $\alpha_{0}$ & $\kappa_{m}$ & $x_{m}$ \\
\hline CT5 & 0.755 & $0.016^{\circ}$ & $2.51^{\circ}$ & 0.0814 & 0.25 \\
\hline \hline
\end{tabular}

Table 1 CT5 Case Parameters. 
and the energy of higher POD modes normally decays rapidly which is evident from Figure 4. Figure 4 also suggests only a limited range of POD modes dominate the flow field, which further implies that proper truncation of POD modes can provide an accurate approximation. With the POD basis extracted, it is possible to obtain the interpolation points using the DEIM, shown in Figure 5. The selection process is only applied along the airfoil surface, then the pressure field is reconstructed by the POD modes with the information at selected DEIM points. The DEIM points cluster near the shock and stagnation area where the pressure gradient is relatively large. With the DEIM points available, the RBF-ANN is used to define the nonlinear mapping between the input trajectory, in this case the aerofoil pitch and the pressure history at the DEIM points. Figure 6 compares the time history of the pressure coefficient obtained from the full-order simulation with the ROM at two locations on the airfoil surface: the first tracking point is near the mid-chord on the upper

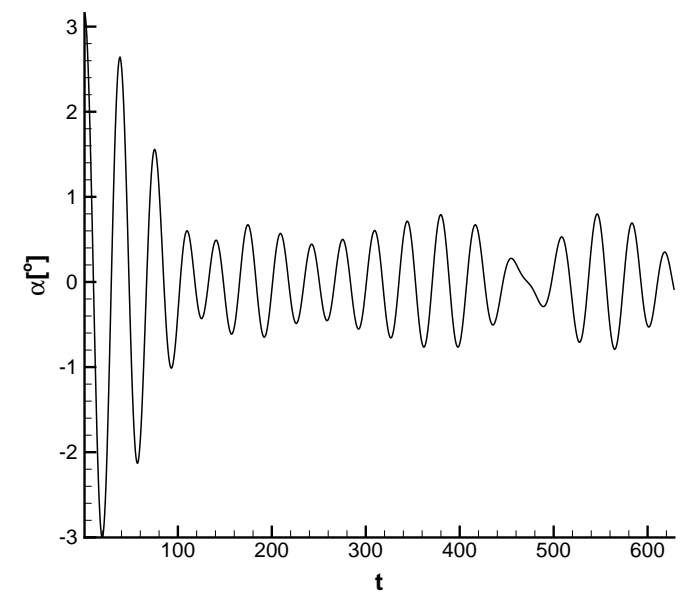

Fig. 2 Training trajectory for forced motion tests.

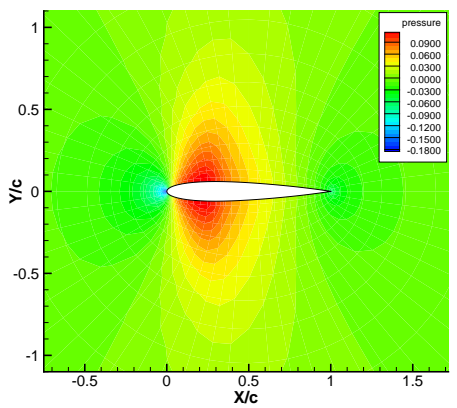

POD Mode 1

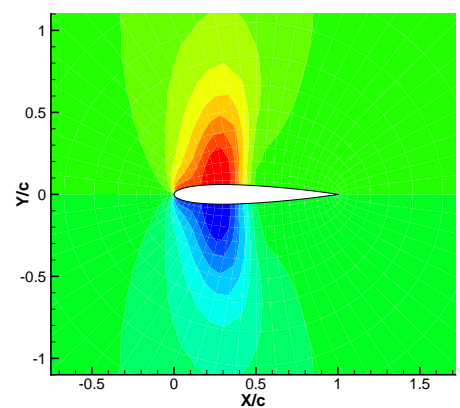

POD Mode 2

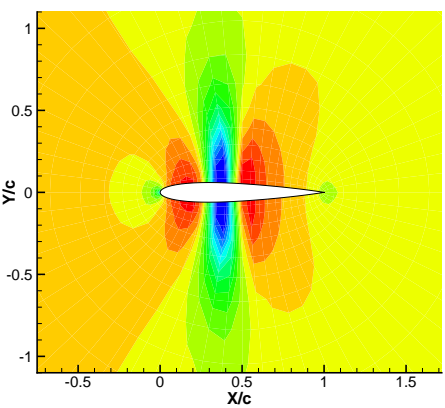

POD Mode 3

Fig. 3 First three pressure POD modes. 
surface, the second is at the trailing edge (all time history plots display one in every four symbols for clarity). The training process aims to define a suitable number of delays to get a satisfactory approximation and maintain a desired level of efficiency. It is expected that the accuracy improves as more delays are added, this however compromises efficiency. Results show that four delays, i.e. $n=4$, suffice to approximate the FOM across the time sequence. At this stage, the pressure field is reconstructed using Eq. (9) with the pressure history predicted by the RBF-ANN, which also allows the aerodynamic forces and moments to be extracted. The surrogate model is complete at this point. Figure 7 shows results using the reconstruction procedure. Regions exhibiting the largest amplitudes are more difficult to recreate and 20 POD modes are necessary to capture the pitching moment accurately. The recreated pressure field and surface pressure coefficient distributions at $t=90$ and $t=400$ are shown in Figures 8-9 together with the FOM reference solution. At time

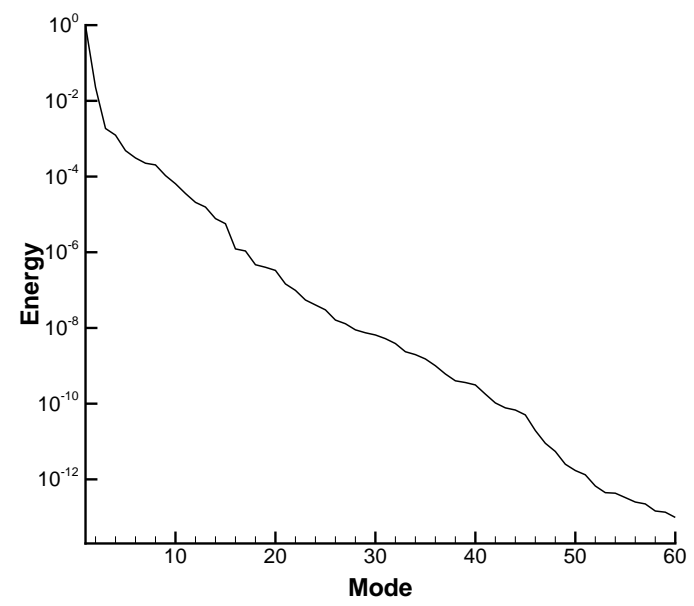

Fig. 4 Snapshot matrix eigenvalue energy decay.

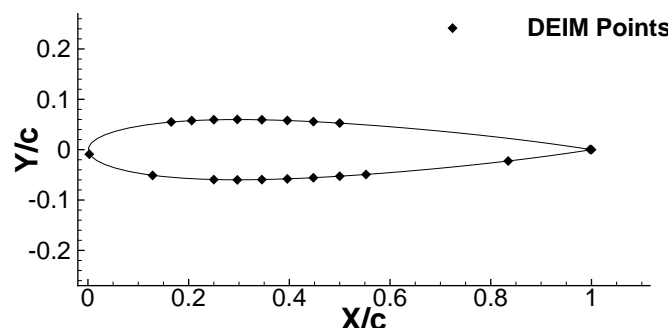

20 DEIM points

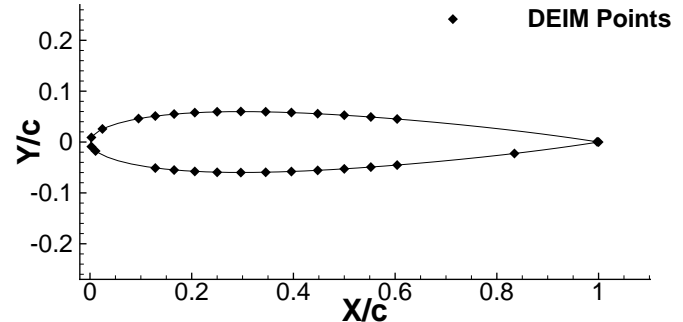

30 DEIM points

Fig. 5 DEIM point distributions. 
point $t=90$, a shock forms on the upper surface, whereas at the second time point a mild shock appears on the lower surface. At both points, the ROM predictions are in excellent agreement with the FOM results, indicating the ROM is able to successfully reconstruct the flow field from the interpolation points. Next, the model is validated for new inputs with different pitching amplitudes and frequencies. The first input corresponds to the CT-5 test case conditions described in Table 1, which are within the training trajectory range of values. The integrated loads cycle as a function of angle-of-attack is shown in Figure 10 which includes numerical and the experimental

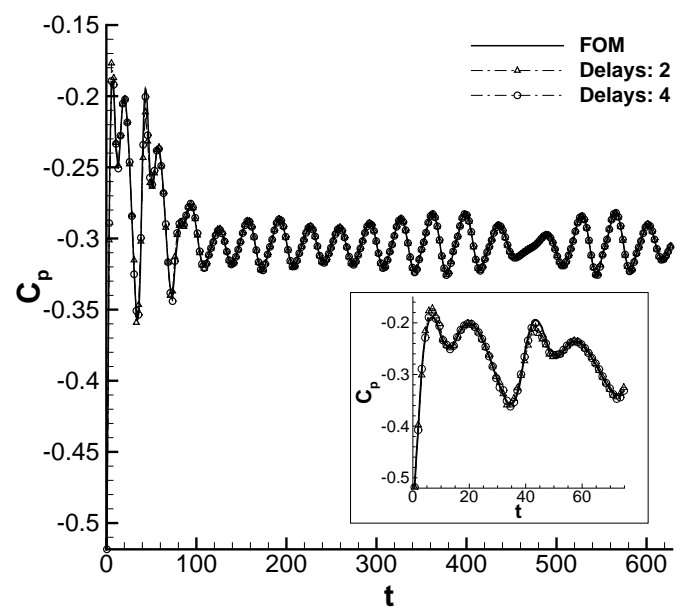

(a) $X / c=0.5$

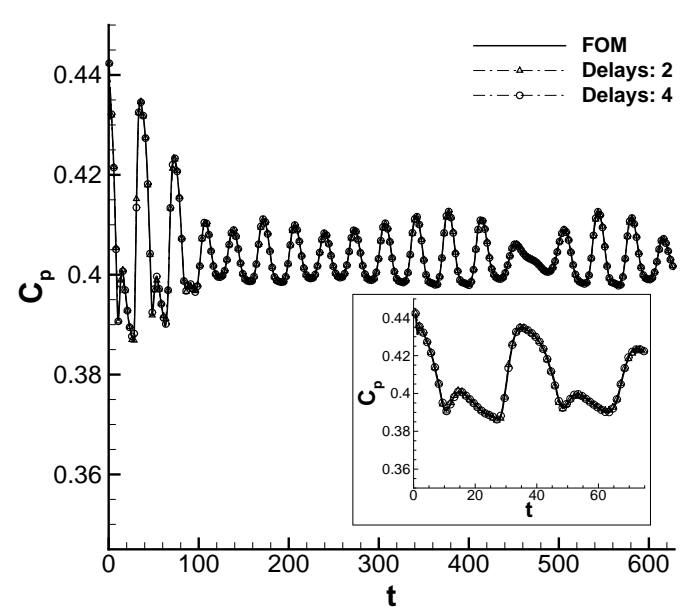

(b) $X / c=1$

Fig. 6 Pressure coefficient time history predicted by the RBF-ANN.

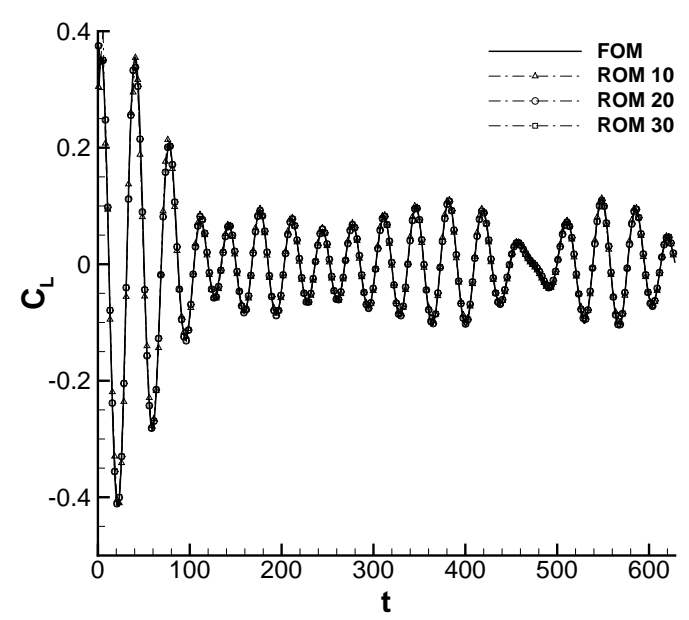

(a)

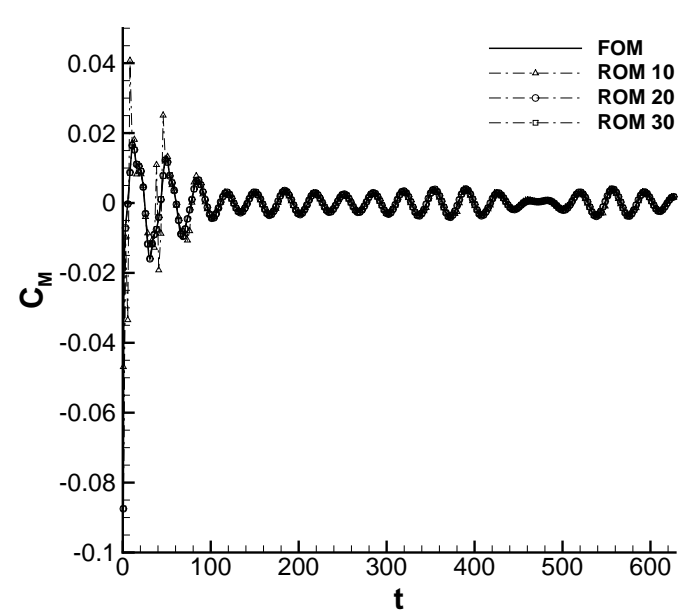

(b)

Fig. 7 Integrated loads time history comparison. 
results. The difference between the inviscid results and the experiment values is consistent with results found in the literature[30, 31]. The ROM slightly under predicts the extreme values for $C_{N}$ by less than $5 \%$ with respect to the FOM, the pitching moment cycle is predicted within a smaller tolerance. The essential features - shock location and intensity - of the flow field of the reference solution are reconstructed by the ROM as illustrated in Figure 11. Another input is defined as $\left[\alpha_{m}, \kappa\right]=\left[1.5^{\circ}, 1.15 \kappa_{m}\right]$. Figures 12 and 13 show consistent agreement with the FOM results. As

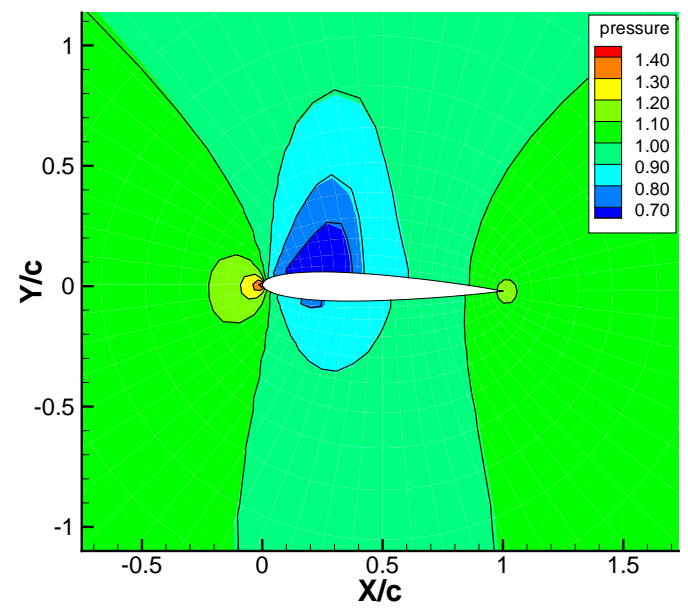

(a) $t=90$

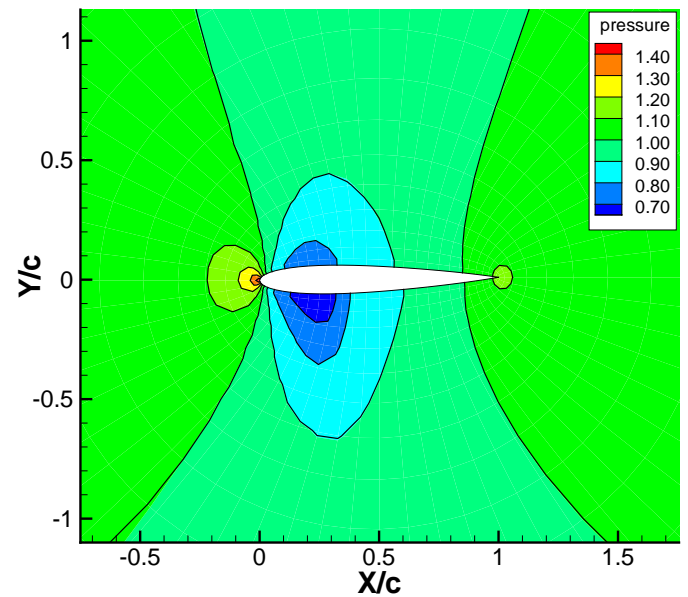

(b) $t=400$

Fig. 8 Pressure flow field - contours represent the CFD results, solid lines correspond to the ROM

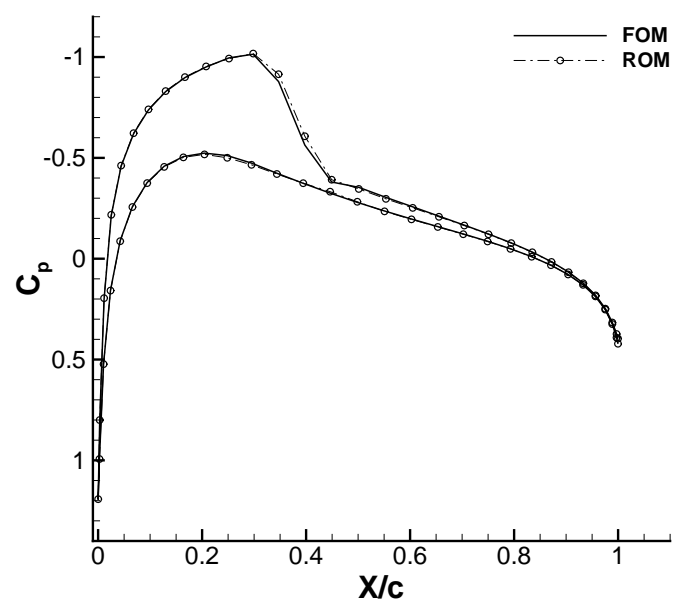

(a) $t=90$

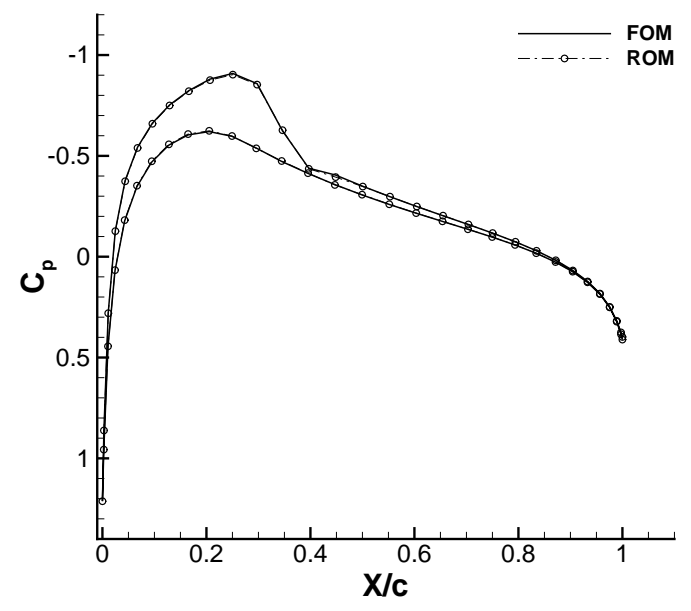

(b) $t=400$

Fig. 9 Surface pressure coefficient comparison. 


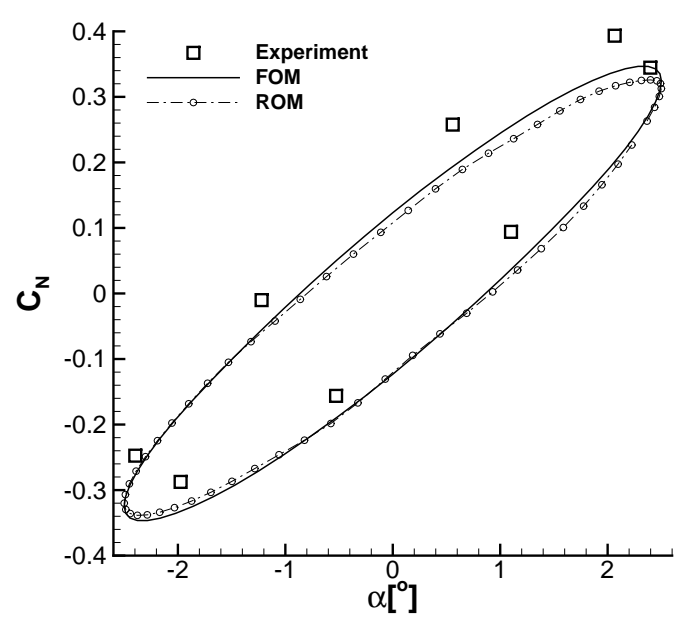

(a) Normal force coefficient

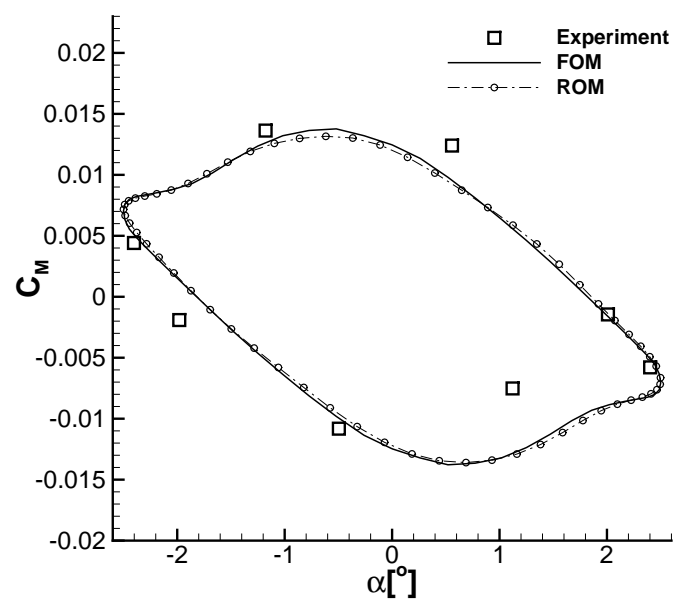

(b) Pitching moment coefficient

Fig. 10 CT-5 normal force and pitching moment coefficients comparison; experiment values obtained from reference[29].

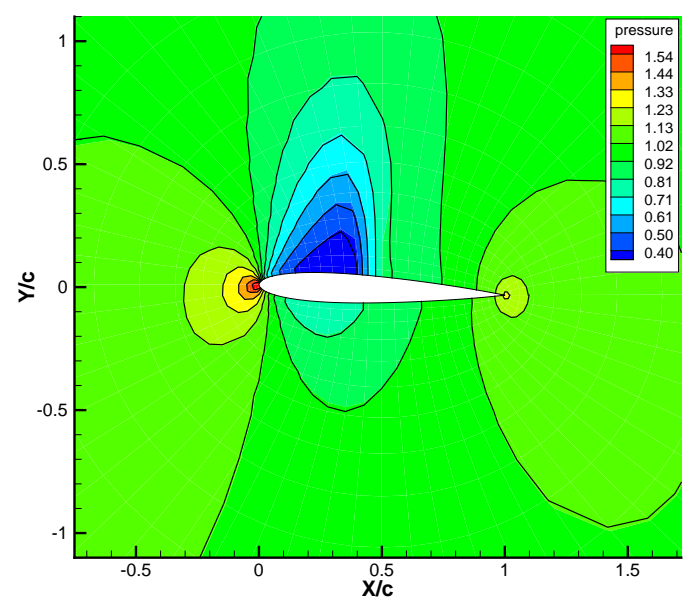

(a) Pressure flow field: contours - FOM; solid lines

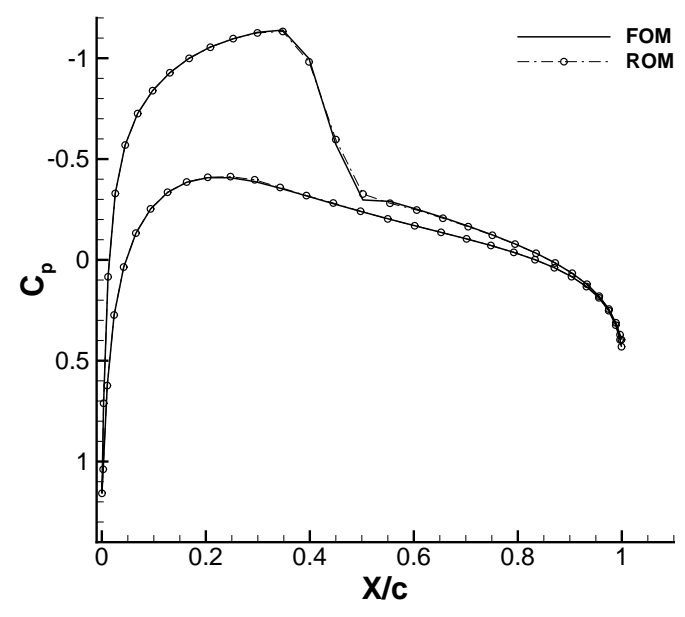

(b) Surface pressure coefficient

- ROM

Fig. 11 Pressure field and corresponding surface pressure coefficient extracted at $\alpha=2.5^{\circ}$ comparison.

in the previous case, the ROM is able to predict the loads correctly and reconstruct the complete flow field from the limited number of interpolation points. Table 2 shows the computational cost associated with the CFD analysis and with the effort required to construct the ROM, which consists of generating the training trajectory, determining the DEIM interpolation points and perform the RBF-ANN identification. As expected, the expensive part of the process is generating the training 


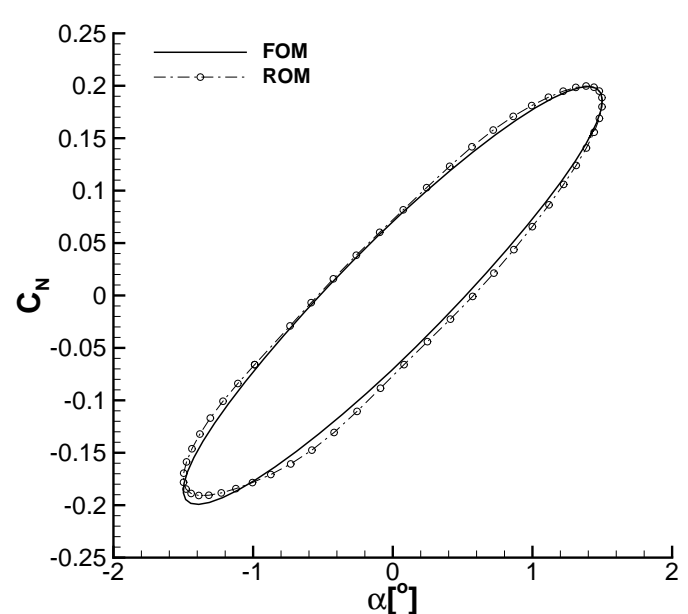

(a) Normal force coefficient

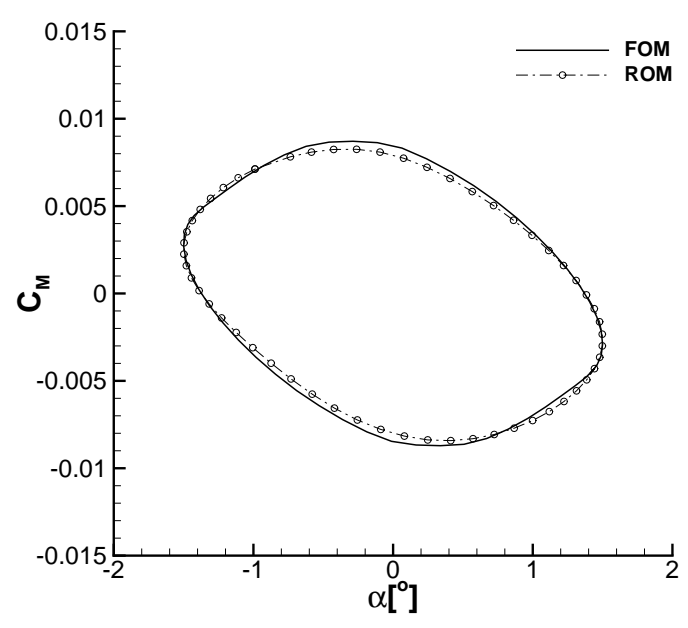

(b) Pitching moment

Fig. 12 Normal and pitching moment coefficients comparison with $\alpha_{m}=1.5^{\circ}, \kappa=0.0936$.

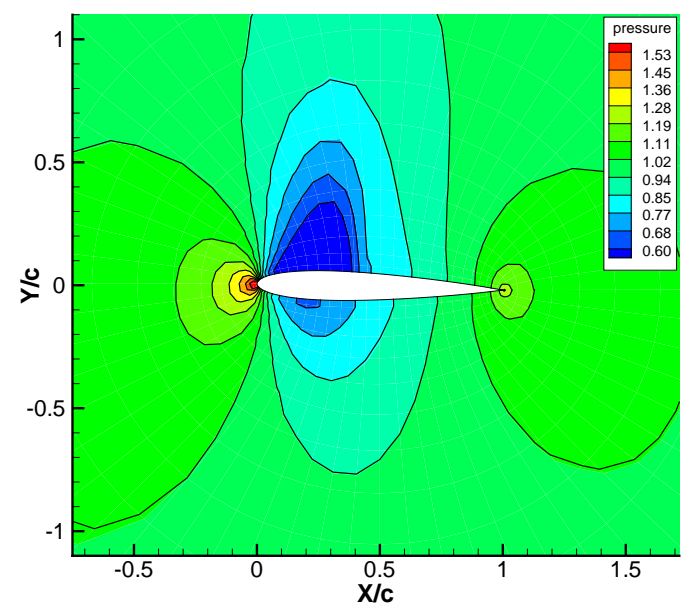

(a) Pressure flow field: contours - FOM; solid lines

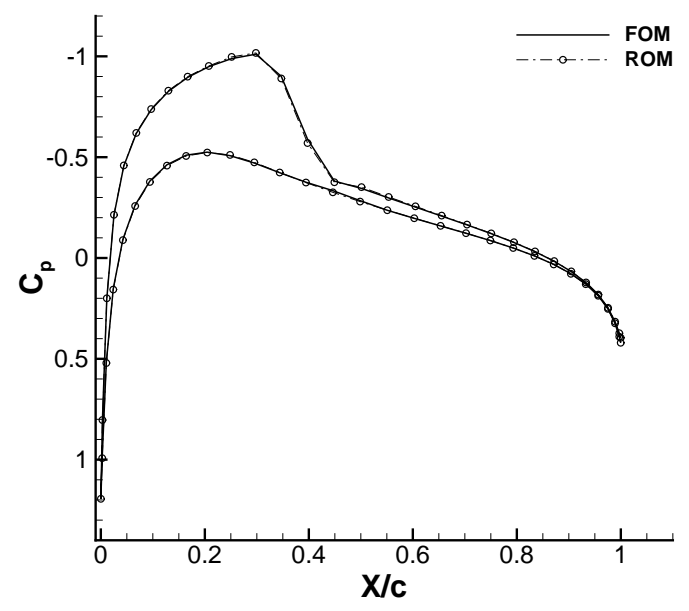

(b) Surface pressure coefficient

- ROM

Fig. 13 Pressure field and corresponding surface pressure coefficient extracted with $\alpha=1.5^{\circ}$ comparison.

data, which requires one time-domain CFD simulation. Then, the ROM replicates the training data at a fraction of the cost of the FOM, a factor of two orders of magnitude in speed-up was observed. The overhead spent in constructing the ROM is spent once. With the model constructed, it can be used for any new input from within the values defined by Eq. (21)-(23), proving the method to be very attractive for conducting parametric studies. 


\begin{tabular}{cccc}
\hline \hline Model & \multicolumn{2}{c}{ Wall clock [minutes] } \\
\hline \multirow{3}{*}{ Training Data DEIM RBF-ANN } \\
Building ROM & 48.2 & 0.1 & 1.8 \\
\hline ROM & 0.3 & & \\
\hline \hline
\end{tabular}

Table 2 Computational effort to build ROM.

\section{B. Two degree-of-freedom aeroelastic system}

In this section, the ability of the ROM to predict the impact of aerodynamic nonlinearities, i.e. shocks, on aeroelastic stability will be investigated by using a pitch/plunge symmetric NACA $64 A 010$ airfoil, as described in reference[3] and shown in Figure 14. The non-dimensional form of

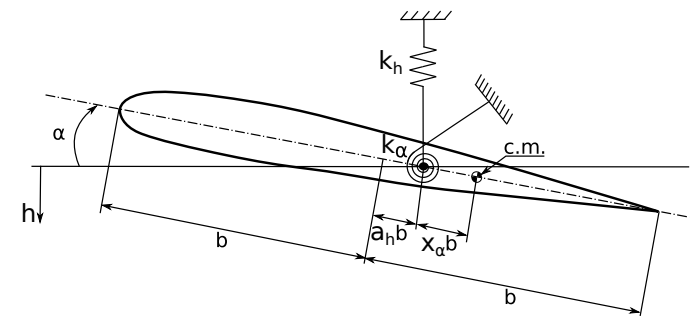

Fig. 14 Diagram of pitch/plunge airfoil system.

the governing equations of motion for a two degree-of-freedom aerofoil can be described as

$$
\mathbf{M} \ddot{\xi}+\frac{1}{\mu V_{s}^{2}} \mathbf{K} \boldsymbol{\xi}=\frac{4}{\pi \mu} \mathbf{f}
$$

where

$$
\mathbf{M}=\left[\begin{array}{cc}
1 & x_{\alpha} \\
x_{\alpha} & r_{\alpha}^{2}
\end{array}\right], \mathbf{K}=\left[\begin{array}{cc}
\left(\frac{\omega_{h}}{\omega_{\alpha}}\right)^{2} & 0 \\
0 & r_{\alpha}^{2}
\end{array}\right], \mathbf{f}=\left[\begin{array}{c}
-C_{l} \\
2 C_{m}
\end{array}\right], \quad \xi=\left[\begin{array}{c}
\frac{h}{b} \\
\alpha
\end{array}\right], \quad V_{s}=\frac{U_{\infty}}{\omega_{\alpha} b \sqrt{\mu}}
$$

the structural parameters are given in Table 3 , where $S_{\alpha}, I_{\alpha}$ are the first and second moment of inertia about the elastic axis, respectively, $m$ is the mass of the airfoil, $\omega_{h}$ and $\omega_{\alpha}$ are the plunge and pitch motion frequencies, respectively.

The Mach number and angle of attack are 0.8 and $0^{\circ}$, respectively. The velocity index, $V_{s}$, is used to set different instability points, i.e. flutter and LCO conditions. Several different methodologies to provide the training trajectory are available from the literature. Lindhorst et al. proposed using 


\begin{tabular}{lc}
\hline \hline Static unbalance, $x_{\alpha}=S_{\alpha} / m b$ & 0.25 \\
Radius of gyration about elastic axis, $r_{\alpha}^{2}=I_{\alpha} / m b^{2}$ & 0.75 \\
Frequency ratio, $\omega_{h} / \omega_{\alpha}$ & 0.5 \\
Mass ratio, $\mu=m / \pi \rho_{\infty} b^{2}$ & 75 \\
\hline \hline
\end{tabular}

Table 3 Pitch/Plunge Aerofoil Parameters.

a prescribed motion together with a frequency module to cover the expected range frequencies[17]. However, it may not be possible to approximate the amplitude and frequency of the LCO a priori. Zhang et al. used a self excited response to step inputs of $V_{s}$, the first input was at a reduced velocity below the flutter threshold, at a subsequent pre-determined point in time, it introduced a $V_{s}$ value within the LCO regime. In this work, the training trajectory is defined using a single input, $V_{s}$, according to

$$
V_{s}=V_{s 0}\left(1-\delta \sin ^{2}(2 \kappa t)\right)
$$

To ensure a suitable range of reduced velocities for this case $V_{s 0}=0.8, \delta=0.15$ and $\kappa=0.015$, this results in the range $0.68 \leq V_{s} \leq 0.8$, which includes the flutter onset velocity, $V_{s}=0.71[4]$, and a suitable range of post-critical velocities. The resultant training trajectory with a relative large plunge perturbation is shown in Figure 15-(a) and the corresponding range of frequencies of the settled down part of the trajectory is illustrated by the Hilbert-Huang Transformation (HHT)[32] in Figure 15-(b). The user's parameters $V_{s 0}, \delta$ and $\kappa$ can be adapted to generate the required training trajectory, as it will be shown in the subsequent test case. Snapshots are collected from the training process and the POD modes extracted following the same procedure described for the forced motion problem. The energy associated with each eigenvalue decays rapidly, as seen in Figure 16-(a). Thirty POD modes are retained and the associated DEIM points used for interpolation are given in Figure 16-(b). The first three POD modes are illustrated in Figure 17. The first mode represents mean flow, the second and the third are POD modes associated with the shock. The inputs to the RBF-ANN are the pitching and plunging displacements at the DEIM points using 4 delays. The ability of the ROM to reconstruct the flow solution is first evaluated by comparing the results for the training trajectory. For both the degrees-of-freedom and airfoil loads, the ROM is able to recreate 
the FOM output, see Figure 18. The ROM is then used to evaluate the system's dynamic response at a series of post-critical $V_{s}$ values. A case near the limit of the training data parameter range, $V_{s}=0.775$, is investigated in detail. At this reduced velocity the system develops an LCO and Figure 19 shows the amplitudes for both plunge and pitch; the ROM systematically under predicts both amplitudes by a small margin, it also shows a small delay in reaching the final amplitude of the LCO, the frequency of the motion is well captured. The overall performance of the ROM can be assessed by examining the LCO amplitude growth in Figure 21, the largest discrepancy is found

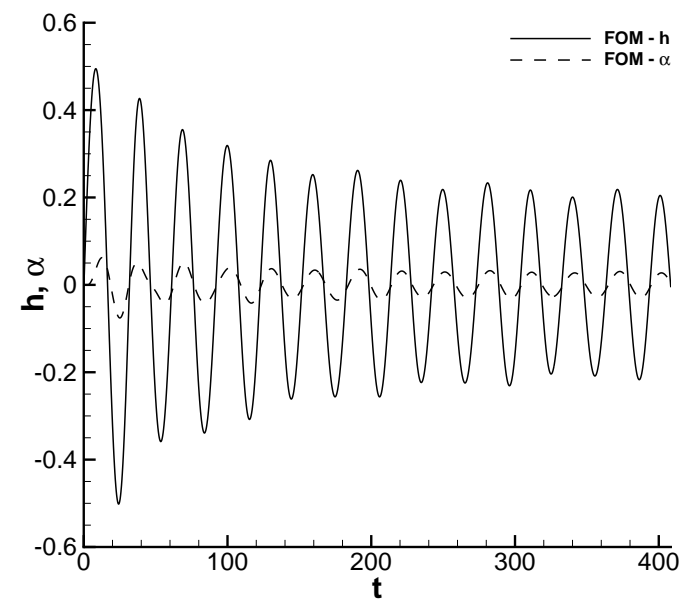

(a) Training trajectory from varying $V_{s}$

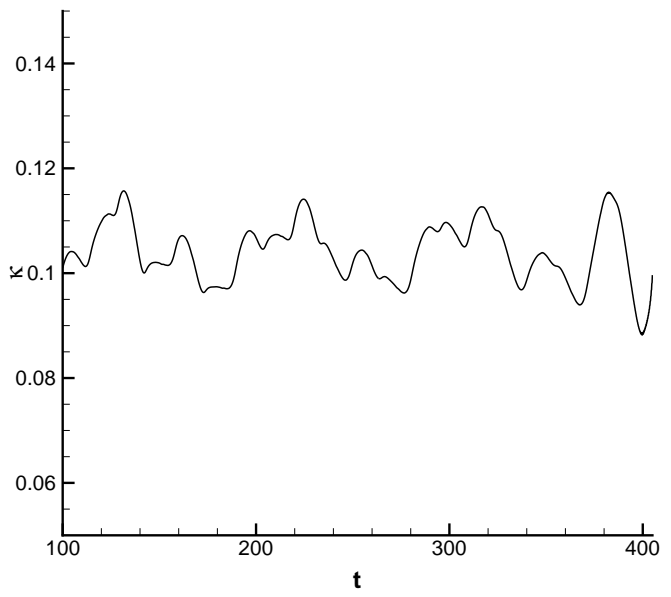

(b) HHT of training trajectory

Fig. 15 LCO training trajectory.

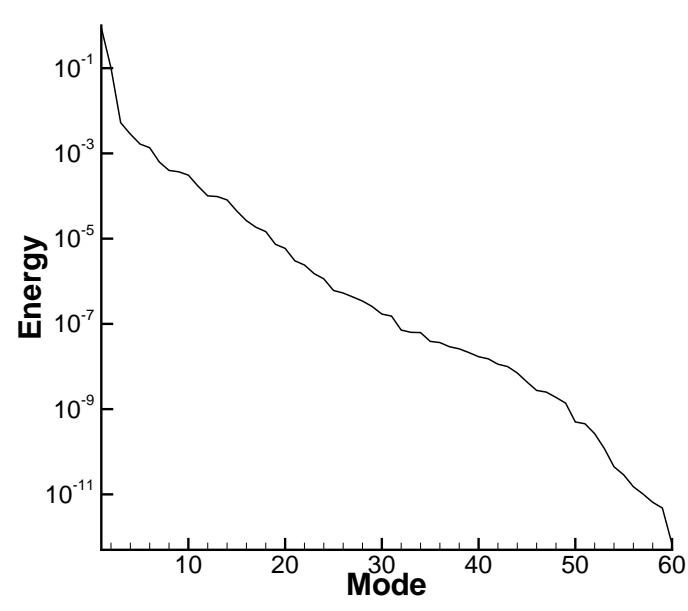

(a) Snapshot matrix eigenvalue decay

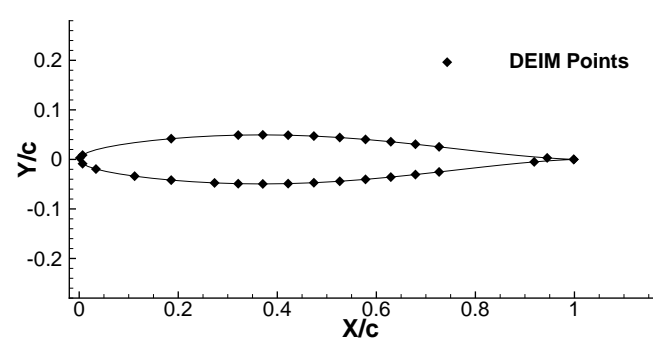

(b) Location of DEIM points

Fig. 16 Snapshot matrix eigenvalue energy and retained DEIM points. 
to be at $V_{s}=0.775$ where the ROM under predicts the full-order simulation by about $5 \%$. The computational effort required to build the ROM is given in Table 4. Once the ROM is built, each calculation above the critical speed requires approximately 20 seconds.

\begin{tabular}{cccc}
\hline \hline Model & \multicolumn{2}{c}{ Wall clock [minutes] } \\
\hline \multirow{3}{*}{ Training Data DEIM } & RBF-ANN \\
Building ROM & 180.5 & 0.12 & 9.95 \\
\hline ROM & 0.32 & & \\
\hline \hline
\end{tabular}

Table 4 Computational effort to build ROM.

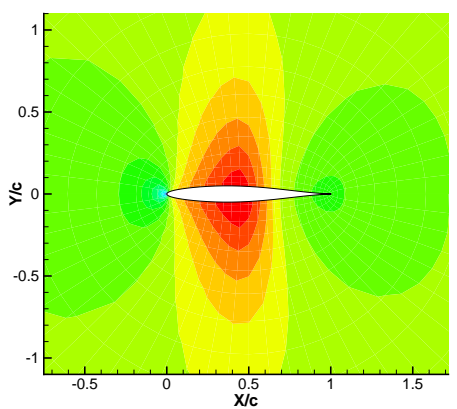

(a) POD Mode 1

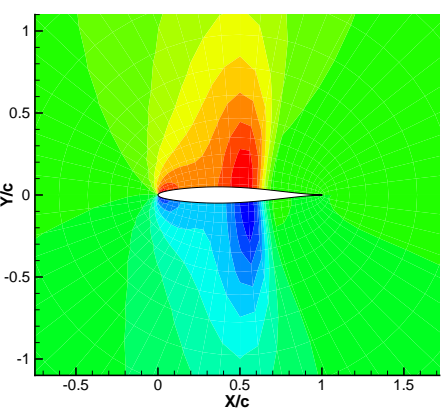

(b) POD Mode 2

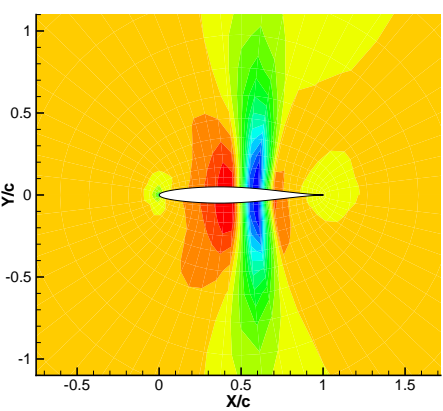

(c) POD Mode 3

Fig. 17 POD modes from pressure flow field.

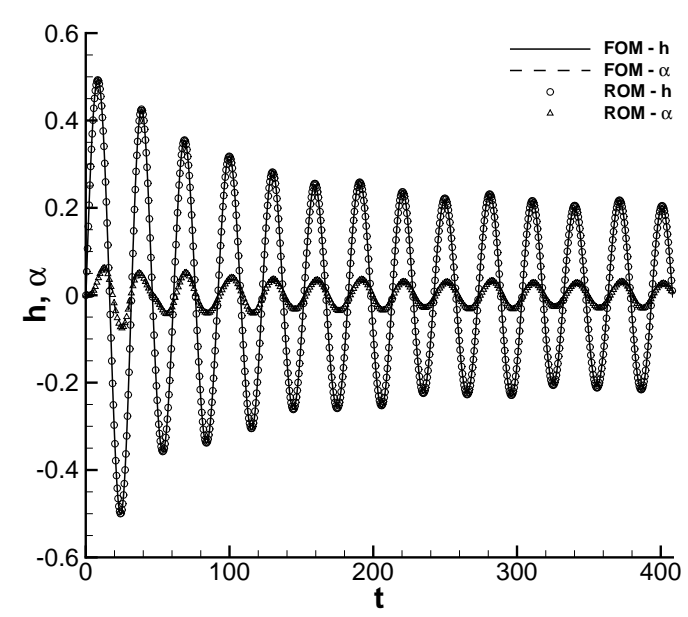

(a) Training trajectory displacements

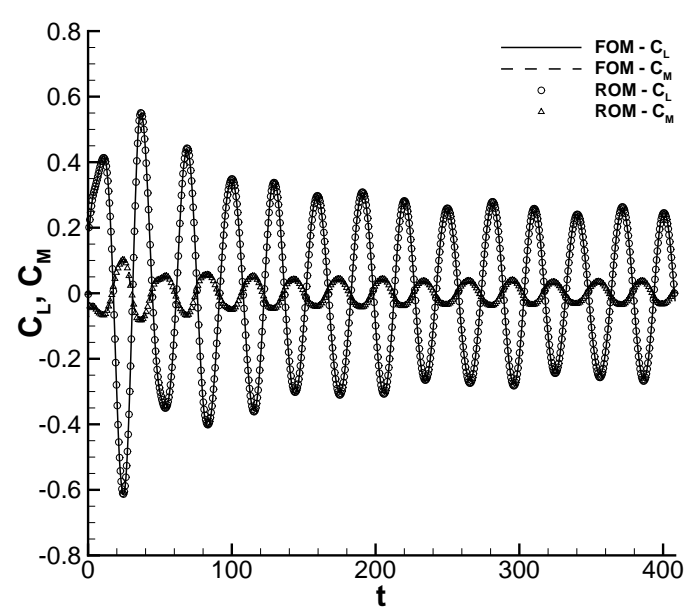

(b) Training trajectory loads

Fig. 18 Training data evaluation. 


\section{Cropped Delta Wing}

To further validate the proposed method, a cropped delta wing model is used; the wing uses a $N A C A 65 A 004$ airfoil, the leading edge sweep angle is approximately $16^{\circ}$ and the semi-span is just under $4 \mathrm{~m}$. The wing structure is modelled using $2 \mathrm{D}$ shell elements and the material properties are based on the AGARD 445.6 wing[33]. Modal analysis performed with MSC/NASTRAN shows the first four modes to be in the frequency range of $4 H z-30 H z$, as illustrated in Figure 22 . The LCO behaviour of the wing has been investigated by time-marching and harmonic balance methods in reference[4], where it was found the wing exhibited LCOs at transonic conditions, Mach number of 0.91 and $0^{\circ}$ angle-of-attack, due to the motion of the shock located near the trailing edge. As before, the ROM first requires building the training trajectory, which in this case is generated by varying the dynamic pressure $q$ as

$$
q=q_{0}\left(1-\delta \sin ^{2}(2 \kappa t)\right)
$$

where $q_{0}=0.873 q_{s l}$ which is $15 \%$ above the flutter onset conditions $\left(q_{f}\right)$ and $q_{s l}$ is the dynamic pressure at sea level conditions, the remainder parameters are: $\delta=0.15$ and $\kappa=0.01$. The final training trajectory obtained is shown in Figure 23-(a), where the coordinate $\eta$ corresponds to the displacement of the wing tip's trailing edge. The HHT results in Figure 23-(b) reveal the range of frequencies covered, ensuring the frequencies of interest are integrated in the training process.

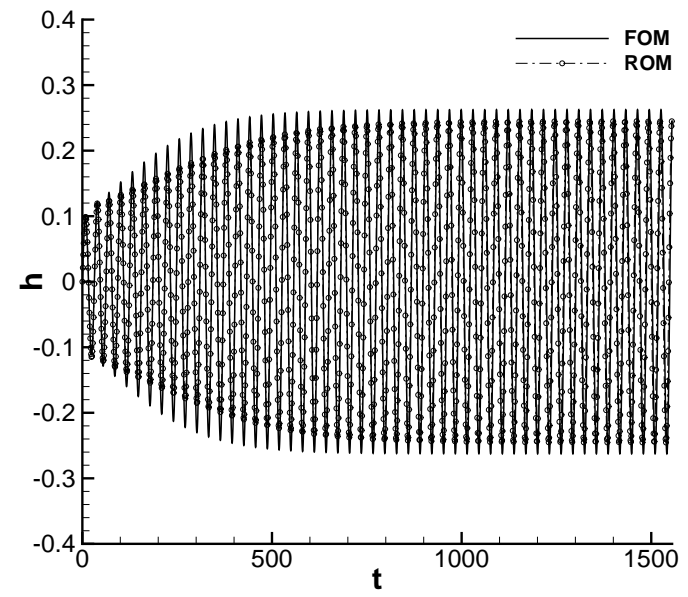

(a) Plunge displacement

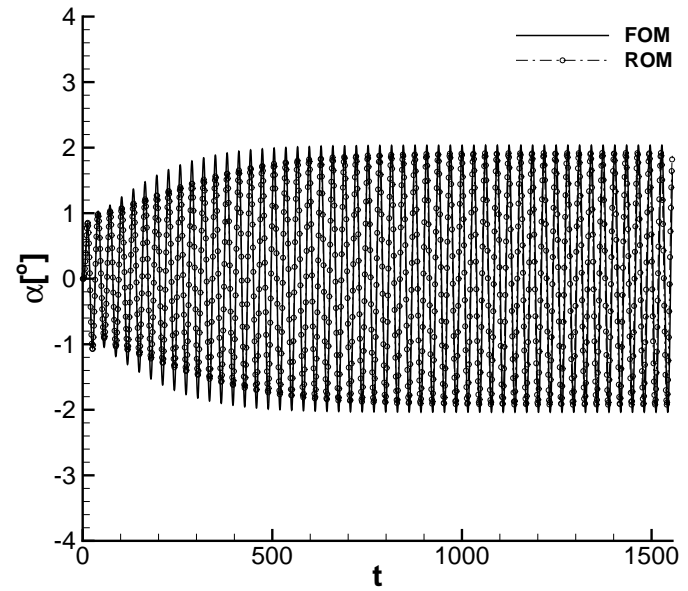

(b) Pitch displacement

Fig. 19 LCO time history comparison of CFD and ROM. 


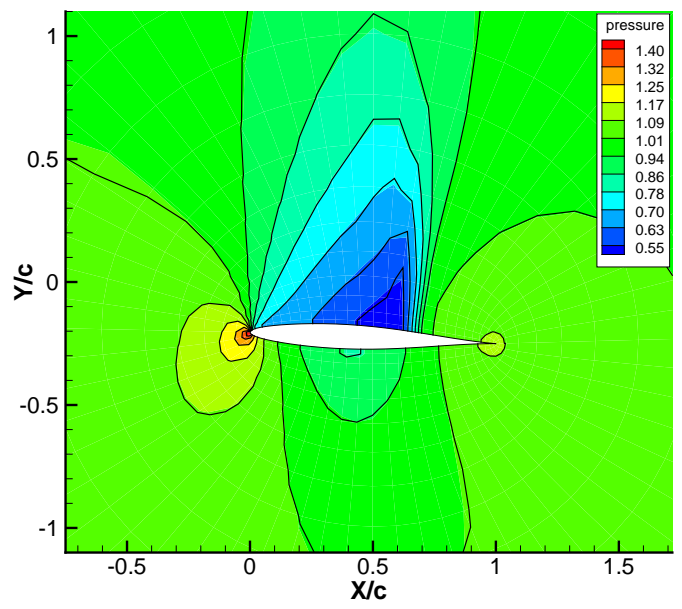

(a) Pressure flow field - contours - FOM; solid lines - ROM

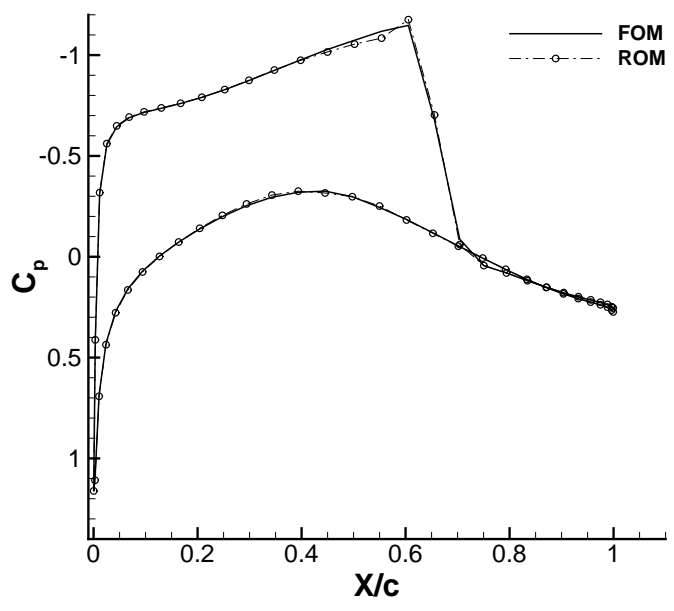

(b) Surface pressure coefficient

Fig. 20 Pressure flow field and surface pressure coefficient at minimum plunge amplitude.

Following the two-dimensional aeroelastic case, POD modes were extracted and the eigenvalue history is shown in Figure 24. The first three POD modes are shown in Figure 25(a)-(c), which reveals the first mode to represent the mean flow, the second and the third modes are associated with the shock-wave. Unlike the previous cases, the third mode is now asymmetric. A total of 120 DEIM points are used in this case, as shown in Figure 25-(d). The points cluster near the shock wave location, and regions of large pressure gradients. Again, at each DEIM point the RBF-ANN was identified through the training process. The input for this case are the modal displacements and eight delays are used to obtain satisfactory approximation to the full-order model. The ROM is then constructed using 120 POD modes. The ability of the ROM to replicate the displacement and loads observed in the training trajectory is shown in Figure 26, both frequency and amplitude are well captured by the ROM. To exercise the ROM at post-critical conditions, the dynamic pressure is set $15 \%$ higher than the flutter condition. As the oscillations develop, during the downwards motion of the wing the shock at the trailing edge moves rewards and a strong suction peak forms on the wing tip, during the upwards movement the shock moves forward and eventually vanishes, the suction peak at the tip moves to the lower surface and the cycle is reversed and restarted. The reconstructed upper surface pressure field as the wing tip bends downwards is compared against the FOM and shown in Figure 27, indicating the proposed methodology is not hindered by the 

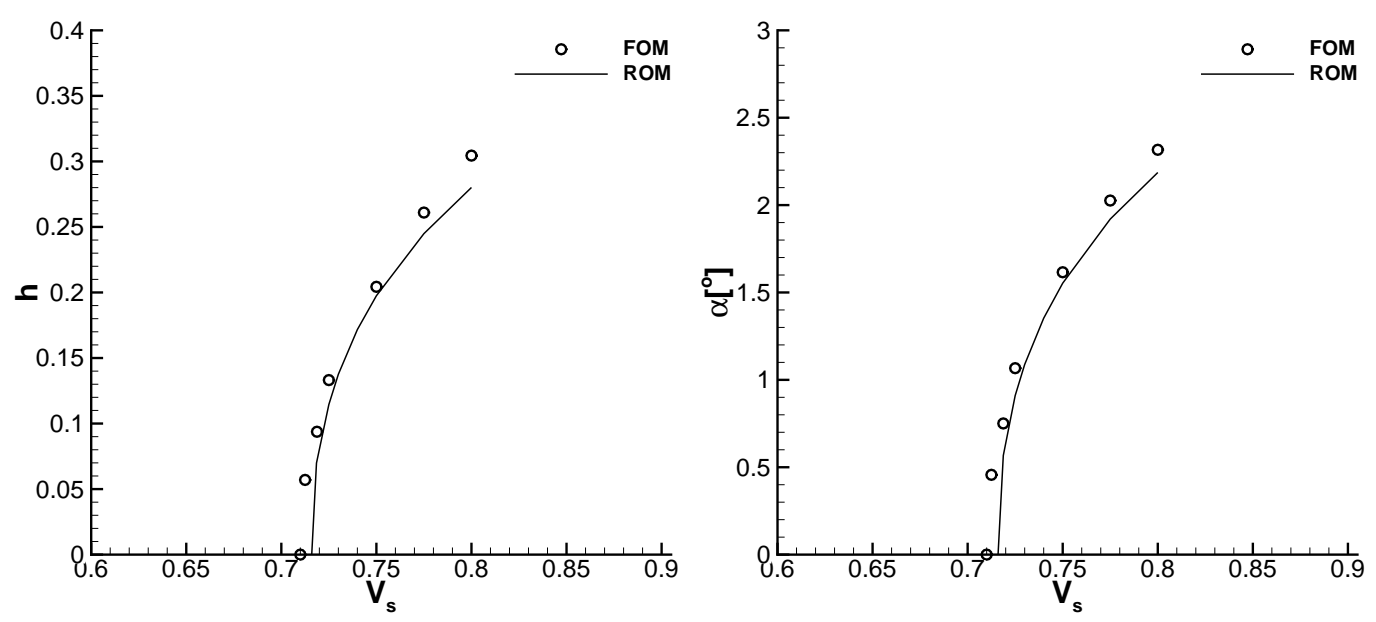

(a) Amplitude vs. velocity index

(b) Amplitude vs. velocity index
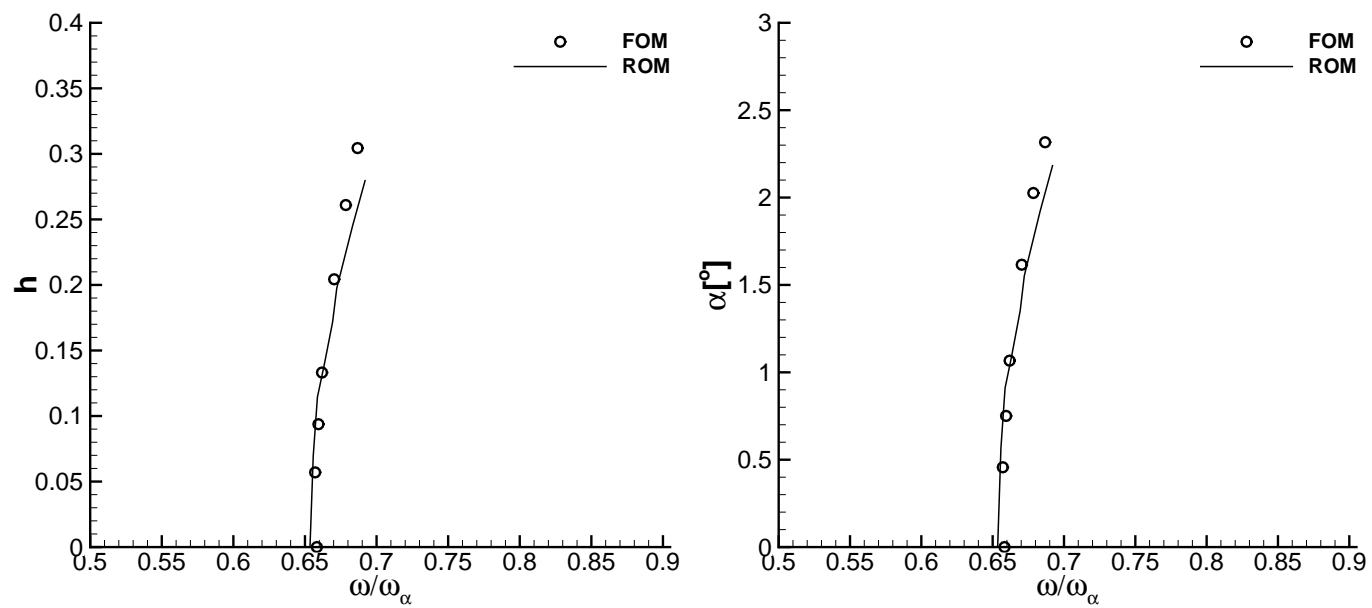

(c) Amplitude vs. frequency ratio

(d) Amplitude vs. frequency ratio

Fig. 21 LCO amplitude comparison between CFD and ROM.

additional complexity of a three-dimensional problem. The LCO mechanism is well predicted by the ROM as demonstrated by the displacement and lift coefficient comparison given in Figure 28 . The evolution of the super-critical LCO until the dynamic pressure reaches $1.15 q_{f}$ is shown in Figure 29 and excellent agreement for both amplitudes and frequencies predicted is observed.

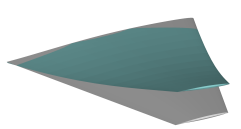

(a) Mode $1-3.94 \mathrm{~Hz}$

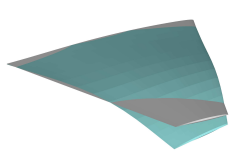

(b) Mode $2-12.88 \mathrm{~Hz}$

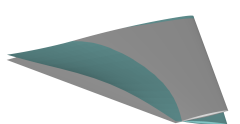

(c) Mode $3-15.82 \mathrm{~Hz}$

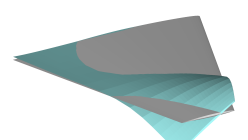

(d) Mode $4-27.56 \mathrm{~Hz}$

Fig. 22 Wing mode shapes and natural frequencies. 


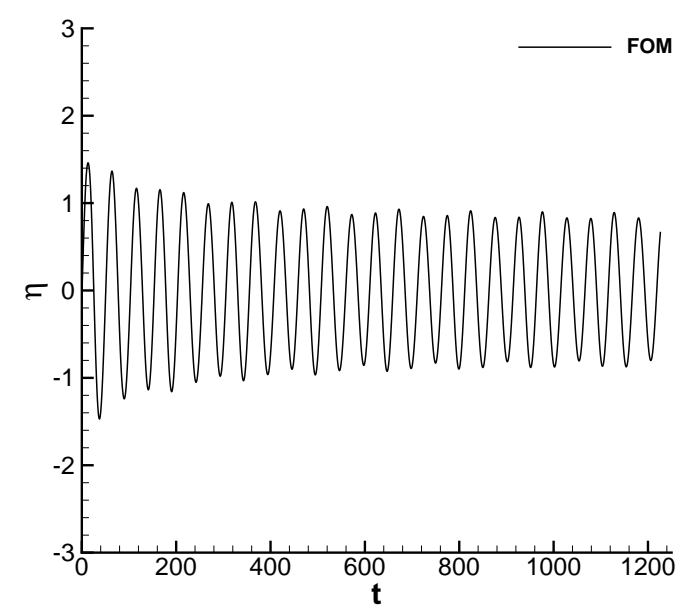

(a) Training trajectory from varying $q$

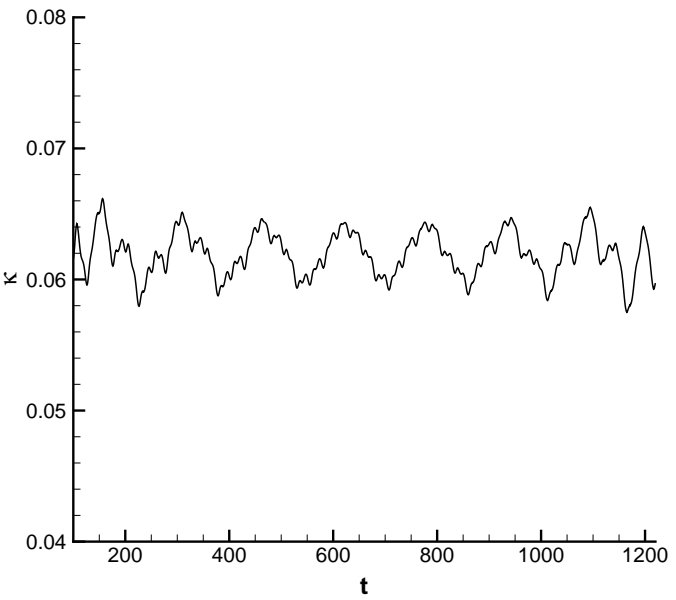

(b) HHT of training trajectory

Fig. 23 LCO training trajectory.

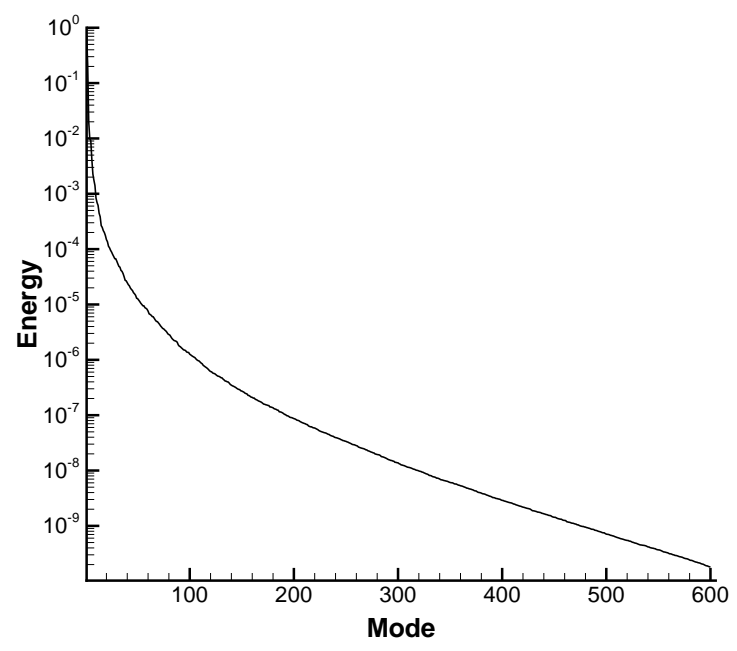

Fig. 24 Snapshot matrix eigenvalue energy decay.

The cost to build and run the ROM is given in Table 5. Each point on the LCO branch takes about one minute to compute using the ROM. The full-order system, using a time step $\Delta t=10^{-5}$ (the time step was selected based on the results from reference[4]), requires about three-orders of magnitude more effort to run than the ROM at $V_{s}=1.15 q_{f}$. It is reasonable to expect this cost to increase at conditions near the flutter onset, where the number of cycles required to reach the limit-cycle tends to increase. 

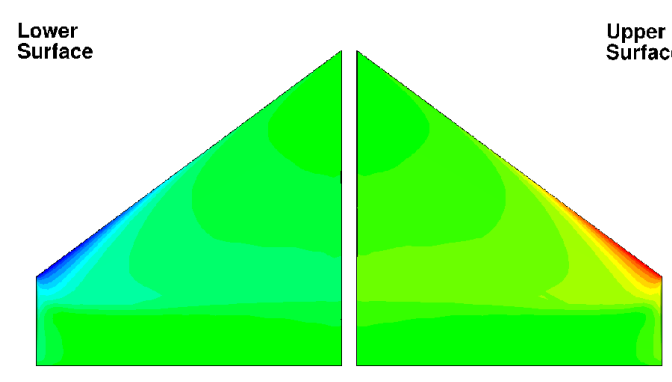

(a) POD mode 1

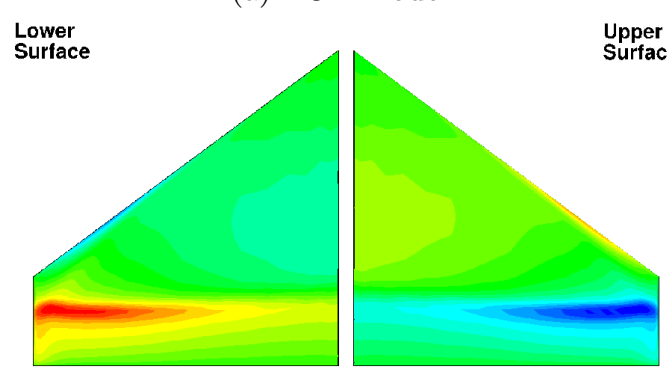

(c) POD mode 3
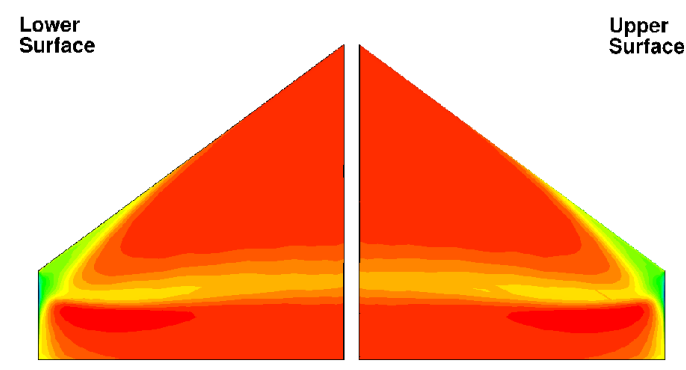

(b) POD mode 2

$\begin{array}{ll}\text { Upper } & \text { Lower } \\ \text { Surface } & \text { Surface }\end{array}$

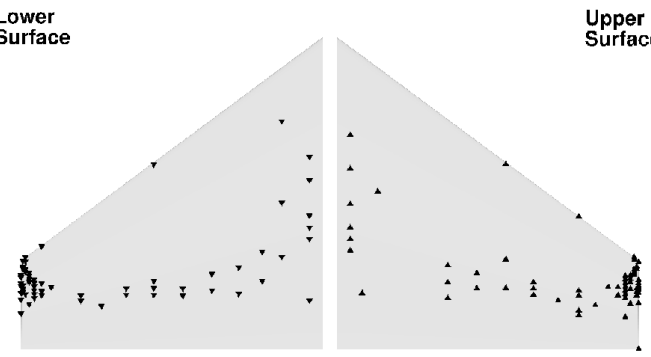

(d) DEIM interpolation points

Fig. 25 POD modes from pressure flow field and DEIM interpolation points.

\section{Conclusions}

A novel surrogate model for nonlinear aerodynamic and aeroelastic simulations was proposed and validated. The novelty of the proposed approach lies in extending the POD/DEIM model reduction technique to problems involving moving boundaries, such as forced motions and LCOs.

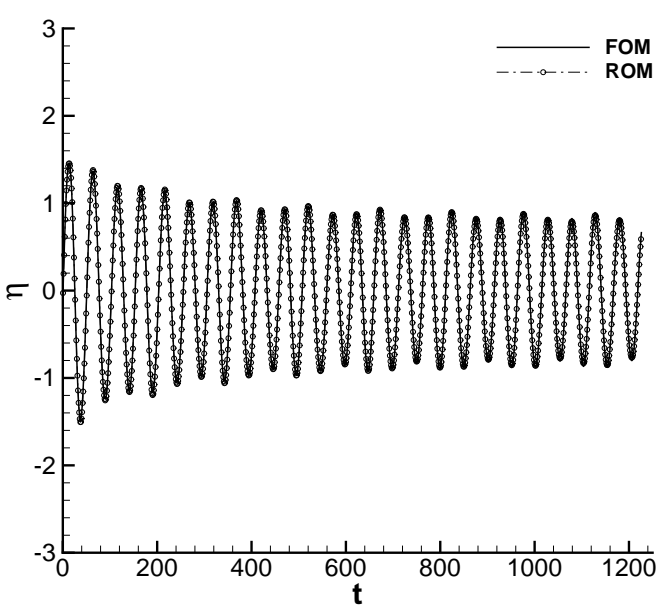

Wing tip displacement

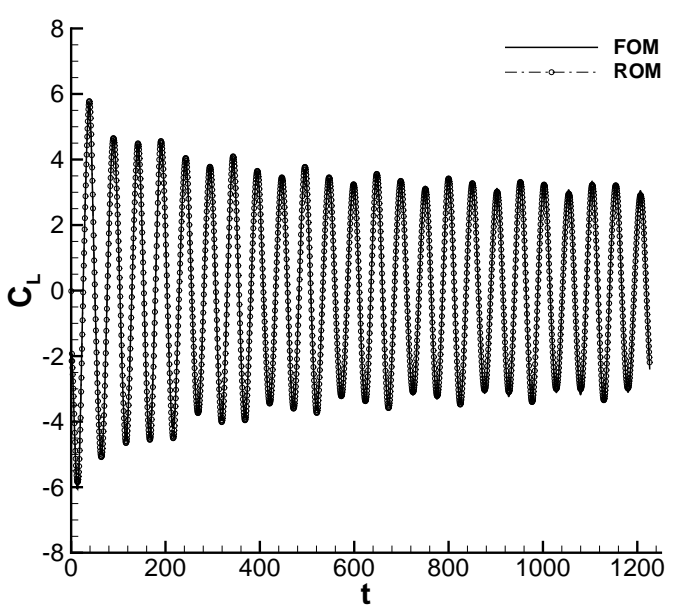

Lift coefficient

Fig. 26 Wing training trajectory comparison. 


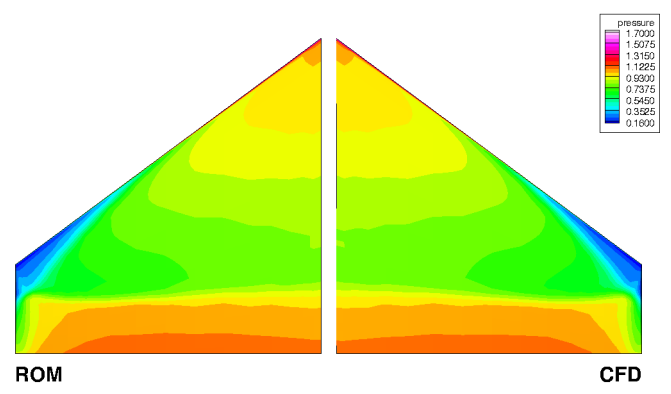

Fig. 27 Upper surface pressure field comparison.

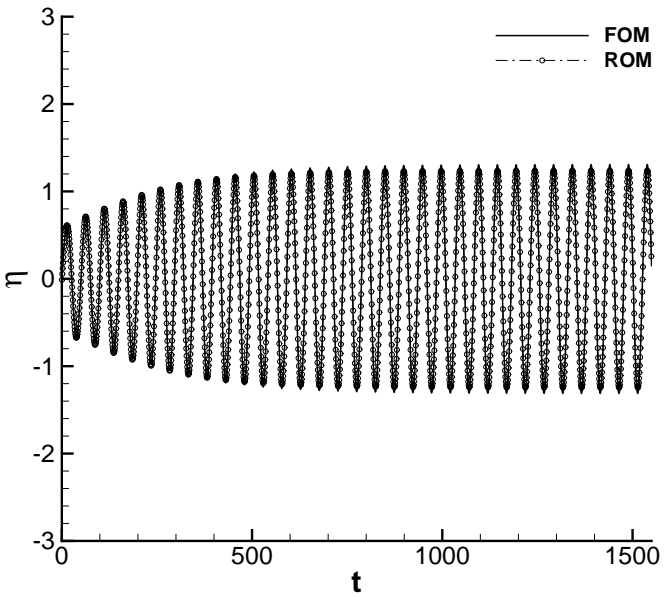

Wing tip displacement

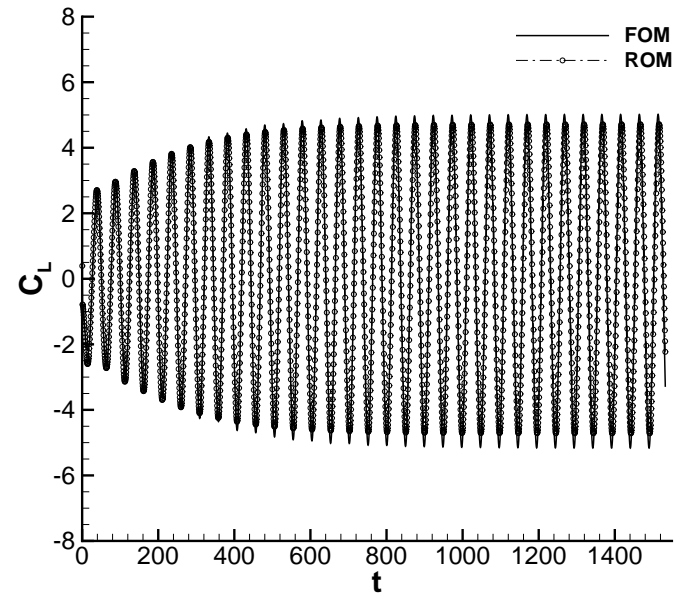

Lift coefficient

Fig. 28 Wing LCO time history

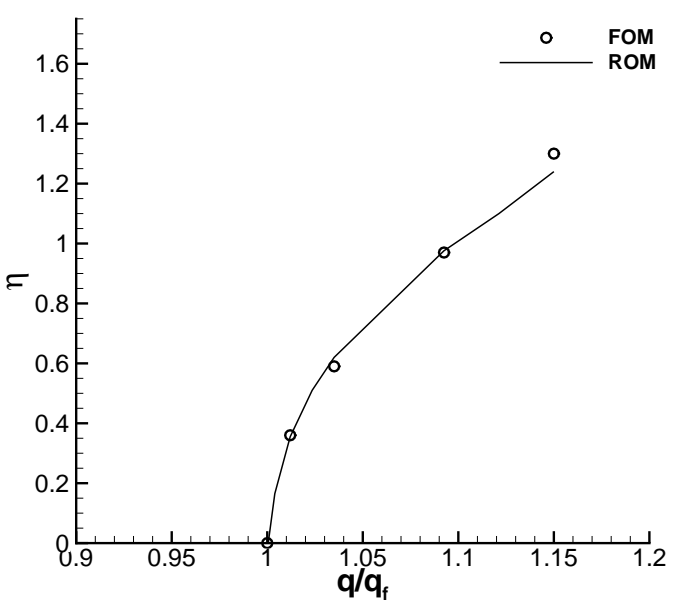

LCO amplitude

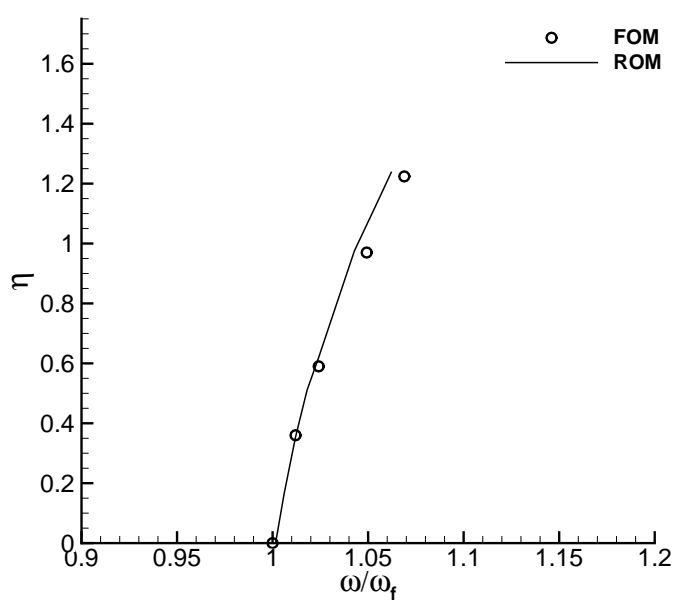

LCO frequency change

Fig. 29 Variation of LCO amplitude and frequency with dynamic pressure.

The new method uses the POD/DEIM to reduce the full-order aerodynamic system to a very limited 


\begin{tabular}{cccc}
\hline \hline Model & \multicolumn{2}{c}{ Wall clock [minutes] } \\
\hline \multirow{4}{*}{ Training Data } & DEIM RBF-ANN \\
Building ROM & 1224 & 0.33 & 88.33 \\
\hline ROM & 1.7 & \\
\hline \hline
\end{tabular}

Table 5 Computational effort to build ROM.

number of POD modes and allows reconstructing the complete flow field using information at key locations on the CFD/CSD interface. This ability allows it to be used as a surrogate on behalf of the CFD solver. An RBF-ANN is used to provide a nonlinear mapping between the structure's displacement and the flow quantity of interest used in the reconstruction of the flow field, at the selected CFD/CSD interface points.

The method was first validated with a $N A C A 0012$ force motion test case, this was followed by a pith/plunge aeroelastic system. Both cases exhibited strong flow nonlinearities in the form of large shock-wave motions. The new ROM was able to capture the main flow features, aerodynamic forces and structural displacements accurately. To highlight the ability of the method in more practical applications, further tests were conducted on a wing geometry, also in the transonic regime. The results obtained further demonstrate the potential of the method to predict LCO amplitudes and frequencies accurately, well beyond the system's bifurcation point.

Ongoing and future work include exploring the DEIM reconstruction procedure for nonlinear aeroelastic problems with additional flow nonlinearities. Problems such as vortex induced vibration and the NLR 7301 test case are currently being investigated.

\section{Acknowledgements}

This work was sponsored by the United Kingdom Engineering and Physical Sciences Research Council (grant number EP/K005863/1). The authors gratefully acknowledged this support.

[1] Badcock, K., Timme, S., Marques, S., Khodaparast, H., Prandina, M., Mottershead, J., Swift, A., Da Ronch, A., and Woodgate, M., Transonic aeroelastic simulation for instability searches and uncertainty analysis, Progress in Aerospace Sciences, Vol. 47, No. 5, (2011), 392-423.

[2] Badcock, K. J., and Woodgate, M. A., Bifurcation Prediction of Large-Order Aeroelastic Models , 
AIAA Journal, vol. 48, No. 6, 2010, 1037-1046.

[3] Thomas, Jeffrey P and Dowell, Earl H and Hall, Kenneth C, Nonlinear inviscid aerodynamic effects on transonic divergence, flutter, and limit-cycle oscillations, AIAA Journal, vol. 40, No. 4, (2002), 638-646

[4] Yao, W., Marques, S., Prediction of Transonic Limit Cycle Oscillations using an Aeroelastic Harmonic Balance Method, AIAA Journal, Vol. 53, No. 7, (2015), pp. 2040-2051. doi: 10.2514/1.J053565

[5] Silva, W., Identification of Nonlinear Aeroelastic Systems Based on the Volterra Theory: Progress and Opportunities, Nonlinear Dynamics, vol. 39, No. 1-2, (2005), 25-62, doi:10.1007/s11071-005-1907-z

[6] Balajewicz, M., and Dowell, E., Reduced-order modeling of flutter and limit-cycle oscillations using the sparse Volterra series, Journal of Aircraft, Vol. 49, No. 6 (2012): 1803-1812.

[7] Hall, K. C., Thomas, J. P., Dowell, E. H., Proper Orthogonal Decomposition Technique for Transonic Unsteady Aerodynamic Flows, AIAA Journal, Vol. 38, No. 10, (2000), 1853-1862., doi: 10.2514/2.867

[8] Thomas, Jeffrey P., Earl H. Dowell, and Kenneth C. Hall. Three-dimensional transonic aeroelasticity using proper orthogonal decomposition-based reduced-order models, Journal of Aircraft, vol. 40, No. 3, (2003), 544-551.

[9] Lucia, D. and Beran, P., Reduced-Order Model Development Using Proper Orthogonal Decomposition and Volterra Theory, AIAA Journal, Vol. 42, No. 6, (2004), pp. 1181-1190.

[10] Lieu, T., Farhat, C., and Lesoinne, M., Reduced-order fluid/structure modeling of a complete aircraft configuration, Computer Methods in Applied Mechanics and Engineering, 195(41), (2006), 5730-5742.

[11] Rowley, C. W., Model reduction for fluids, using balanced proper orthogonal decomposition, International Journal of Bifurcation and Chaos, vol. 15, No. 3, (2005), 997-1013.

[12] Willcox, K. and Peraire, J., Balanced model reduction via the proper orthogonal decomposition, AIAA Journal, Vol. 40, No. 11, (2002), 2323-2330.

[13] Fagley, C., Seidel, J., Siegel, S. and McLaughlin, T., Reduced order modeling using proper orthogonal decomposition (pod) and wavenet system identification of a free shear layer. In Active Flow Control II (pp. 325-339). Springer Berlin Heidelberg, (2010).

[14] Zhang, W., Wang, B., Ye, Z. and Quan, J., Efficient method for limit cycle flutter analysis based on nonlinear aerodynamic reduced-order models, AIAA Journal, vol. 50, No.5, (2012), 1019-1028.

[15] Glaz, B., Liu, L. and Friedmann, P. P., Reduced-order nonlinear unsteady aerodynamic modeling using a surrogate-based recurrence framework, AIAA Journal, vol. 48, No. 10, (2010), 2418-2429.

[16] Mannarino, A., Mantegazza, P., Nonlinear aeroelastic reduced order modeling by recurrent neural networks, Journal of Fluids and Structures, Vol. 48, (July 2014), 103-121. doi: 10.1016/j.jfluidstructs.2014.02.016 
[17] Lindhorst, K., Haupt, M. C. and Horst, P., Efficient surrogate modelling of nonlinear aerodynamics in aerostructural coupling schemes, AIAA Journal, vol. 52, No. 9, (2014), 1952-1966.

[18] Lindhorst, K., Haupt, M. C. and Horst, P., Aeroelastic Analyses of the High-Reynolds-NumberAerostructural-Dynamics Configuration Using a Nonlinear Surrogate Model Approach, AIAA Journal, Vol. 53, No. 9, (2015), pp. 2784-2796. doi: 10.2514/1.J053743

[19] Chaturantabut, Saifon, and Danny C. Sorensen, Nonlinear model reduction via discrete empirical interpolation, SIAM Journal on Scientific Computing, vol. 32, No. 5, (2010), 2737-2764.

[20] Carlberg, K., Farhat, C., Cortial, J. and Amsallem, D., The GNAT method for nonlinear model reduction: effective implementation and application to computational fluid dynamics and turbulent flows. Journal of Computational Physics, 242, (June 2013), 623-647

[21] Yao, W., and Liou, M.-S., A nonlinear modeling approach using weighted piecewise series and its applications to predict unsteady flows Journal of Computational Physics, Vol. 31, No. 8, 2016, pp. 58-84

[22] Xiang, C., Existence of global input-output model for nonlinear systems, In Control and Automation, 2005. ICCA'05. International Conference on. Vol. 1, pp. 125-130, (2005), IEEE.

[23] Liou, M. S., A sequel to AUSM, Part II: AUSM+-up for all speeds, Journal of Computational Physics, vol. 214, No. 1, (2006), 137-170.

[24] Van Leer, B., Towards the ultimate conservative difference scheme. II. Monotonicity and conservation combined in a second-order scheme, Journal of computational physics, vol. 14, No. 4, (1974), 361-370.

[25] Timme, S.,Marques, S. and Badcock., K., Transonic aeroelastic stability analysis using a kriging-based Schur complement formulation, AIAA Journal, vol.49, No. 6, (2011): 1202-1213.

[26] Benner, P., Gugercin, S. and Willcox, K., A survey of projection-based model reduction methods for parametric dynamical systems. SIAM Review vol. 57, No. 4, (2015): 483-531.

[27] Nguyen, N. C., and Peraire, J., An efficient reducedorder modeling approach for nonlinear parametrized partial differential equations, International Journal for Numerical Methods in Engineering, vol. 76, No. $1,(2008), 27-55$.

[28] Orr, Mark, Introduction to radial basis function networks., Center for Cognitive Science, University of Edinburgh, Edinburgh TR, Scotland, U.K., 1996.

[29] Landon, R. H., NACA 0012. Oscillating and Transient Pitching, In Compendium of Unsteady Aerodynamic Measurements, AGARD R-702, Data Set 3, 1982

[30] Batina, John T, Unsteady Euler Airfoil Solutions Using Unstructured Dynamic Meshes, AIAA Journal, vol. 28, No. 8, (1990), 1381-1388. 
[31] Da Ronch, A., McCracken, A., Badcock, K., Widhalm, M. and Campobasso, M., Linear frequency domain and harmonic balance predictions of dynamic derivatives, Journal of Aircraft, vol. 50, No. 3, (2013), 694-707

[32] Huang, N. E. and Wu, Z., A review on HilbertHuang transform: Method and its applications to geophysical studies, Reviews of Geophysics, vol. 46, No. 2, (2008), doi:10.1029/2007RG000228.

[33] Yates, E. C., AGARD standard aeroelastic configurations for dynamic response I-wing 445.6, (No. AGARD-R-765), Advisory Group For Aerospace Research And Development, (1987), Neuilly-Sur-Seine (France) 